\title{
TRAVELLING WAVES IN PHASE FIELD MODELS OF SOLIDIFICATION
}

\author{
MICHAEL N. BARBER ${ }^{1}, 2$ and DAVID SINGLETON ${ }^{1,3}$
}

(Received 4 August 1992; revised 18 February 1993)

\begin{abstract}
The existence and selection of steady-state travelling planar fronts in a set of typical phase field equations for solidification are investigated by a combination of numerical and analytical methods. Such solutions are conjectured to exist only for a unique velocity of propagation and to be unique except for translation. This behaviour is in marked contrast to the situation in conventional Stefan models in which travelling fronts exist for all velocities. The value of the steady-state velocity depends upon the various material parameters which enter the phase field equations. Numerical and, in certain tractable limits, analytical results for the velocity are presented for a number of physical situations.
\end{abstract}

\section{Introduction}

Conventional approaches to modelling a solidification front usually involve the assumption that the front is a sharp mathematical surface separating the solid and liquid phases. If it is further assumed that this surface is the level set $\Gamma$ of the temperature field $T(\mathbf{x}, t)$ on which the temperature equals the melting temperature, $T_{M}$, we obtain a classical Stefan problem [36]. In this formulation $T(\mathbf{x}, t)$ satisfies the heat equation in both phases, while conservation of energy implies that the normal velocity of the front $\Gamma$ is

$$
, v_{n}=\frac{c_{s} D}{L} n \cdot\left[(\nabla T)_{\text {solid }}-(\nabla T)_{\text {liquid }}\right] \text {, }
$$

where $(\nabla T)_{\text {solid/iquid }}$ are the limiting values of the gradient as the interface is approached from the solid and liquid, respectively and $n$ is the normal to $\Gamma$ directed from the solid to liquid phase. The parameters $D, L, c_{s}$ are material constants - respectively, the diffusion constant, the latent heat and the specific heat. While the Stefan problem in

\footnotetext{
'Department of Mathematics, Australian National University, ACT, Australia.

${ }^{2}$ Pro Vice-Chancellor (Research), The University of Western Australia, Nedlands, WA, Australia.

${ }^{3}$ ANU Supercomputer Facility, Australian National University, ACT, Australia.

(C) Australian Mathematical Society, 1994, Serial-fee code 0334-2700/94
} 
this simplest form has received considerable attention and continues to do so, important physics is neglected. The most significant physical omissions are the effects of surface tension, interfacial thickness, metastability and kinetic effects.

Surface tension effects can be included by modifying the boundary condition applied at the (sharp) interface to include the Gibbs-Thompson correction. Mathematically, this modification replaces the simple specification of the front as a level set of the temperature field by the condition that

$$
T_{\text {front }}=T_{M}\left(1-\frac{\sigma \kappa}{L}\right),
$$

where $\sigma$ is the surface tension and $\kappa$ is the principal curvature of the front. Physically, the derivation of the Gibbs-Thompson correction assumes local thermodynamic equilibrium in the vicinity of the interface [25]. This modification of the classical Stefan problem forms the basis of current theories of velocity and morphology selection in dendritic growth. (For reviews, see $[27,22,30]$.)

The question of metastability cannot be adressed quite so easily, since specifiying the temperature $T(\dot{\mathbf{x}}, t)$ at a particular point $\mathbf{x}$ does not identify the phase (usually, equilibrium solid or metastable, supercooled liquid) existing at $\mathbf{x}$. To do so, we need to introduce a second field $\phi(\mathbf{x}, t)$, which takes different values in the two phases. In statistical mechanical terms, $\phi$ is essentially an order parameter. However, in this context it is usually referred to as the phase field.

The existence of a spatially varying order parameter is also the essential ingredient of statistical mechanical descriptions of equilibrium interfaces between coexisting phases; see, for example [35, 21]. In these theories, which have been extensively developed and refined over the past twenty years or so, the interface is no longer a sharp mathematical surface but an interfacial region in which the order parameter varies rapidly. The success of these theories in understanding the behaviour of equilibrium interfaces and related phenomena suggests that the incorporation of similar concepts in dynamic situations, that are at least in quasi-equilibrium, could be advantageous. Phase field models are a natural way to do this since they are built on an assumption of local thermodynamic equilbrium.

In this paper we discuss and analyse in some detail one aspect of the behaviour of phase field models of solidification, namely the existence and selection of planar travelling waves. The paper is organized as follows. In the next section, we briefly review the physical basis and derivation of phase field models focussing on those aspects of particular relevance to our considerations. Section 3 similarily summarizes the relevant features and results of the statistical mechanical theory of an equilbrium interface. Section 4 specializes the phase field equations to the case of a steadystate planar front. These steady-state equations form the basis of a series of detailed numerical and analytical calculations that are reported in Sections 5 to 10. The paper 
closes with an overall summary in Section 11, where we also link our work more closely to other related recent work. Some technical aspects are relegated to a series of appendices.

\section{Phase field models}

Detailed derivations ${ }^{4}$ of phase field models have been given by Penrose and Fife [31] and by Caginalp and Jones [8]. Such considerations are beyond the scope of this paper. It suffices to recall the basic ideas.

The dynamical equations we require can be 'derived' from an appropriate local thermodynamic free energy functional $\mathcal{F}=\mathcal{F}[\phi, u, \ldots]$ of the phase field and any other relevant physical fields. ${ }^{5}$ For our purposes only the temperature is necessary, which we introduce as the reduced field

$$
u=\left(T-T_{M}\right) T_{M}=u(x, t),
$$

where $T_{M}$ is the bulk melting temperature. The essential and crucial thermodynamic features are captured if we take $\mathcal{F}$ to be of the form

$$
\mathcal{F}[\phi, u, \ldots]=\int d x\left\{\frac{1}{2} K(\nabla \phi)^{2}+\Psi(\phi)-\alpha u \phi-\frac{1}{2} \beta u^{2}\right\},
$$

where $\Psi(\phi)$ is assumed to have two equal minima at $\phi= \pm 1$. The parameters $K, \alpha, \beta$ can be expressed in terms of equilibrium material constants $[26,11]$. For the simple functional (2.2), such considerations give

$$
\alpha=L / 2 k_{B} T_{M}, \quad \beta=c_{s} / k_{B},
$$

where $k_{B}$ is Boltzmann's constant, while $K$ can be related to the surface tension, see (3.3) below.

Dynamically, the phase field is governed by a Landau-Ginzburg equation:

$$
\tau \phi_{t}=-\delta \mathcal{F} / \delta \phi,
$$

where $\tau$ is a relaxation time constant. The functional derivative is defined by the condition that

$$
\left.\frac{d}{d s} \mathcal{F}[\phi+s \chi]\right|_{s=o}=\int d x \chi(x) \frac{\delta \mathcal{F}}{\delta \phi}(x)
$$

\footnotetext{
${ }^{4}$ More heuristic or physically motivated derivations are given in $[26,11,4]$, while Wheeler et. al. [38] have constructed a precise phase field model to describe an isothermal phase transition in a binary alloy. ${ }^{5}$ We absorb a normalization factor of $k_{B} T_{M}$, where $k_{B}$ is Boltzmann's constant, into the definition of $\mathcal{F}$. The physical dimensional free energy is thus $k_{B} T_{M} \mathcal{F}$.
} 
holds for all test functions $\chi(x)$. Explicitly evaluating the functional derivative for the form (2.2) yields:

$$
\tau \phi_{t}=K \nabla^{2} \phi-\Psi^{\prime}(\phi)+\alpha u
$$

for the first of our basic equations.

The second equation is just energy conservation, which implies that

$$
T s_{t}=\nabla \cdot q_{T},
$$

where $s$ is the entropy density and $q_{T}$ is a thermal current. The entropy follows from $\mathcal{F}[\phi, u]$ by the usual thermodynamic relation $s / k_{B}=-\delta \mathcal{F} / \delta u$, while we assume that $q_{T}$ is given by Fourier's law

$$
q_{T}=D c_{s} \nabla T,
$$

with $D$ the diffusion constant and $c_{s}$ the specific heat. With these assumptions (2.7) reduces to

$$
(1+u)\left[\alpha \phi_{t}+\beta u_{t}\right]=k_{B} D c_{s} \nabla^{2} u,
$$

which is the second basic equation.

The function $\Psi(\phi)$ has not until now been specified except for the condition that it has two equal minima at $\phi= \pm 1$. Most of our ensuing analysis will require only the further condition that $\Psi^{\prime \prime}(\phi)>0$ for $\phi$ near \pm 1 . More precisely we will require the following condition to hold. Consider the equation

$$
\Psi^{\prime}(\phi)+\Delta=0
$$

where $\Delta$ is a non-negative constant. Then we assume, for sufficiently small $\Delta$, that $(2.10)$ possesses three roots

$$
-1 \sim \bar{\phi}_{-1}<\bar{\phi}_{0}<\bar{\phi}_{+1} \sim+1,
$$

such that

$$
\Psi^{\prime \prime}\left(\bar{\phi}_{-1}\right)>0 \text { and } \Psi^{\prime \prime}\left(\bar{\phi}_{+1}\right)>0 .
$$

For more specific calculations, including all our numerical work, we will use:

$$
\Psi(\phi)=\left(\phi^{2}-1\right)^{2} / 8
$$

We shall refer to this choice as " $\phi^{4}$-theory" since the resulting free energy functional $\mathcal{F}$ is then the familar functional of $\phi^{4}$-field theory.

Equation (2.9) is not quite as simple as that usually assumed in the literature. To recover this equation it is necessary to assume that $u$ is small and neglect the explicit $u$ 
dependence on the left-hand side of (2.9). If we do this and, in addition, scale $u \rightarrow \alpha u$ and $x \rightarrow x \sqrt{\beta k_{B} / D c_{s}},(2.6)$ and (2.9) reduce to the system

$$
\begin{aligned}
\tau \phi_{t} & =\epsilon^{2} \nabla^{2} \phi-\Psi^{\prime}(\phi)+u, \\
u_{t}+\lambda \phi_{t} & =\nabla^{2} u .
\end{aligned}
$$

The new parameters $\epsilon$ and $\lambda$ are given in terms of the original material constants by

$$
\lambda=\alpha^{2} / \beta, \quad \epsilon=\sqrt{K \beta k_{B} / c_{s} D} .
$$

Physically, $\epsilon$ and $\tau$ are both expected to be small.

The system (2.14) and (2.15), or simple variants, has been the basis of almost all theoretical work on phase field models, notably the extensive work of Caginalp and co-workers $[4,7,9]$. In particular, this work has addressed questions of existence, uniqueness and regularity $[4,9]$ and explored the conditions under which various sharp interface limits can be recovered $[5,9,6]$. These equations will similarly form the basis of the detailed work reported here. We return to the question of whether the approximations involved, particularly the simplification of (2.9), are qualitatively significant in our concluding comments in Section 11.

\section{Equilibrium interface}

If we assume that $u$ is everywhere zero and that $\phi$ is independent of $t$, then (2.6) and (2.9) reduce to the single equation

$$
K \nabla^{2} \phi-\Psi^{\prime}(\phi)=0,
$$

which is simply the Euler-Lagrange equation following from the requirement that $\phi(\mathbf{x})$ is an extremum of the free energy functional (2.2) with $u=0$.

The trivial constant solutions $\phi= \pm 1$ correspond to the two bulk homogeneous phases. We shall refer to a phase with $\phi \approx-1$ as 'solid' and one with $\phi \approx+1$ as 'liquid'. Of more interest are solutions that asymptotically approach these bulk values and thus can be identified with interfaces between coexisting phases. These solutions form the basis of the mean-field theory of equilibrium interfacial phenomena; see, for example, [35, 21].

Three consequences of this theory are of importance to our present discussion. Firstly, as already noted, the interface is no longer a sharp mathematical surface but an interfacial region characterized by a rapid variation of $\phi$. For a planar interface, (3.1) reduces to the ordinary differential equation

$$
K \frac{d^{2} \phi}{d x^{2}}-\Psi^{\prime}(\phi)=0
$$


which is to be solved subject to the boundary conditions $\phi \rightarrow \pm 1, \phi^{\prime} \rightarrow 0$ as $x \rightarrow \pm \infty$. The excess free energy associated with this spatially varying extremum, relative to that of a uniform homogeneous bulk phase, is related to the surface tension $\sigma$ of the interface. Specifically, $\sigma$ is given by the Cahn-Hilliard relation $[10,35]$

$$
\sigma / k_{B} T_{M}=K \int_{-\infty}^{\infty}\left[\phi^{\prime}(x)\right]^{2} d x .
$$

For $\phi^{4}$-theory the relevant solution of (3.2) is the single kink solution

$$
\phi_{\text {kink }}=\tanh \left[\left(x-x_{0}\right) / 2 \sqrt{K}\right] \text {, }
$$

where $x_{0}$ is an arbitrary constant that reflects the translational invariance of the position of the interface. Evaluating the integral in (3.3) for this solution gives

$$
\sigma / k_{B} T_{M}=2 \sqrt{K} / 3
$$

which reveals that the parameter $K$, and hence $\epsilon$ in (2.14), is determined in terms of the surface tension. Thirdly, and perhaps less well-known, the Gibbs-Thompson effect is automatically included; the conventional result (1.2) following from a consideration $[26,4]$ of the effect of curvature on the planar kink solution.

\section{Steady-state planar fronts}

We now specialize the system defined by $(2.14)$ and (2.15) to steady-state onedimensional planar fronts moving with constant velocity $c$. Such fronts are represented by solutions of the form

$$
\phi(x, t)=\hat{\phi}(x-c t), \quad u(x, t)=\hat{u}(x-c t),
$$

where the functions $\hat{\phi}$ and $\hat{u}$ satisfy the coupled ordinary differential equations:

$$
\begin{aligned}
\epsilon^{2} \hat{\phi}^{\prime \prime}+\tau c \hat{\phi}^{\prime}+f(\hat{\phi})+\hat{u} & =0 \\
\hat{u}^{\prime \prime}+c\left(\hat{u}^{\prime}+\lambda \hat{\phi}^{\prime}\right) & =0
\end{aligned}
$$

subject to appropriate boundary conditions. Primes denote differentiation with respect to the single variable $\xi=x-c t$ and we have defined

$$
f(\phi)=-\Psi^{\prime}(\phi) .
$$

To specify the boundary conditions recall that we wish to model a planar soldification front moving into a supercooled metastable liquid phase. Far from the front in 
either phase the local temperature should thus approach a constant value that is below the melting temperature. Consequently we assume that

$$
\hat{u}(\xi) \rightarrow-\Delta_{ \pm} \text {as } \xi \rightarrow \pm \infty,
$$

where $\Delta_{+} \geq \Delta_{-} \geq 0$. The requirement, that to the left of the front equibrium solid exists while to the right of the front is metastable liquid, is met if

$$
\hat{\phi}(\xi) \rightarrow \phi_{ \pm} \text {as } \xi \rightarrow \pm \infty
$$

where $\phi_{+}$and $\phi_{-}$satisfy

$$
f\left(\phi_{ \pm}\right)=\Delta_{ \pm}
$$

with $\phi_{+} \sim+1$ and $\phi_{-} \sim-1$. Finally, we impose no flux boundary conditions at infinity ${ }^{6}$, i.e. $\hat{\phi}^{\prime} \rightarrow 0$ and $\hat{u}^{\prime} \rightarrow 0$ as $\xi \rightarrow \pm \infty$.

Integrating (4.3) and applying the boundary conditions as $\xi \rightarrow-\infty$ implies

$$
\hat{u}^{\prime}=-c\left(\hat{u}+\Delta_{-}+\lambda\left(\hat{\phi}-\phi_{-}\right)\right) .
$$

Alternatively applying the boundary conditions as $\xi \rightarrow+\infty$ yields

$$
\hat{u}^{\prime}=-c\left(\hat{u}+\Delta_{+}+\lambda\left(\hat{\phi}-\phi_{+}\right)\right) .
$$

Hence if $\hat{\phi}$ and $\hat{u}$ are to be continuous functions with continuous derivatives for all $\xi \in(-\infty, \infty)$ and $\hat{u}^{\prime} \rightarrow 0$ as $\xi \rightarrow \pm \infty$, we require

$$
\Delta_{+}-\Delta_{-}=\lambda\left(\phi_{+}-\phi_{-}\right) \text {. }
$$

This condition is the analogue of the familar condition [27] that the Stefan number must equal unity for planar steady-state fronts to exist in the classical Stefan problem. Presumably if this condition is relaxed the phase field equations exhibit similar problems of finite-time blow up, etc. [19], although we have not explored this aspect. We shall henceforth assume that (4.10) is satisfied, which implies through the conditions (4.7) that the boundary data $\phi_{ \pm}$and $u_{ \pm}$are all determined in terms of one free parameter which we take to be $\Delta_{-}$.

Within $\phi^{4}$-theory, $f(\phi)=\frac{1}{2} \phi\left(1-\phi^{2}\right)$ so that the equation

$$
f(\phi)=\Delta
$$

with $\Delta$ a non-negative constant is a cubic with at most three real roots. For $\Delta<$ $1 / 3 \sqrt{3}$, the roots are all real and can be written

$$
\bar{\phi}_{k}(\Delta)=\frac{2}{\sqrt{3}} \cos \left(\theta(\Delta)+\frac{2 \pi k}{3}\right), \quad k=-1,0,+1,
$$

${ }^{6}$ For simplicity, we will henceforth usually omit explicit mention of this condition in the statement of the boundary conditions applied to any particular differential equation. 
where

$$
\theta(\Delta)=\frac{1}{3} \cos ^{-1}(-3 \sqrt{3} \Delta)-\frac{2 \pi}{3} .
$$

Hence from (4.7) and (4.10) the required boundary data are explicitly defined in terms of $\Delta_{-}$by

$$
\begin{aligned}
& \phi_{-}=\bar{\phi}_{-1}\left(\Delta_{-}\right)=\frac{2}{\sqrt{3}} \cos \left(\theta\left(\Delta_{-}\right)-\frac{2 \pi}{3}\right), \\
& \phi_{+}=\bar{\phi}_{+1}\left(\Delta_{+}\right)=-\frac{1}{2} \phi_{-}+\sqrt{1-2 \lambda-\frac{3}{4} \phi_{-}^{2}}
\end{aligned}
$$

and

$$
\Delta_{+}=\frac{1}{2} \phi_{+}\left(1-\phi_{+}^{2}\right) \text {. }
$$

Note that $\phi_{-}<0$ and $\phi_{+}>0$ and that to ensure $\phi_{+}$is real, $\lambda$ and $\phi_{-}$must satisfy

$$
\lambda<\frac{1}{2}-\frac{3}{8} \phi_{-}^{2} \text {. }
$$

This condition can be interpreted as either a restriction on $\lambda$ or, rather more naturally, as a restiction on $\Delta_{-}$. However, we do not believe that the restriction has much physical significance and is an artifact of the simplistic structure of the usual phase field equations.

\section{The limit $\epsilon=\tau=0$}

Since $\epsilon$ and $\tau$ are physically both small, the obvious first step is to set both parameters to zero. However, it is also obvious from the structure of (4.2) that this is likely to significantly affect the solution since the limit $\epsilon$ and $\tau$ to zero is singular. We will see that this is indeed the case: In particular, the solution is no longer continuous. Nevertheless the resulting solution is important for two reasons. Firstly, it is qualitatively the same as that obtained from a simple classical Stefan problem and thus allows us to understand the effect of finite interface thickness on the Stefan problem. Secondly, the limit is a necessary mathematical step towards the inclusion of non-zero values of $\epsilon$ and $\tau$ in the analysis.

With $\epsilon=\tau=0,(4.2)$ reduces to the algebraic equation

$$
f(\phi)+u=0,
$$

where henceforth we omit the carets on $\phi$ and $u$. Integrating (4.3) as in the previous section, we augment (5.1) with a first-order ordinary differential equation for $u$ which we write as

$$
u^{\prime}= \begin{cases}-c\left(u+\Delta_{-}+\lambda\left(\phi-\phi_{-}\right)\right) & \text {if } \xi<\xi_{0} \\ -c\left(u+\Delta_{+}+\lambda\left(\phi-\phi_{+}\right)\right) & \text {if } \xi>\xi_{0}\end{cases}
$$


where $\xi_{0}$ is arbitrary. For $\xi>\xi_{0}, \phi(u(\xi))$ is given by the solution of (5.1) that is closest to $\phi=+1$ while for $\xi<\xi_{0}$, we take the solution which is closest to $\phi=-1$. However, unlike the situation with non-zero $\epsilon$, we can no longer maintain continuity of $\phi$ and $u^{\prime}$ across $\xi=\xi_{0}$ even with the condition (4.10) on the boundary data.

Consider $\xi>\xi_{0}$. Eliminating $u$ gives a separable first-order differential equation for $\phi$ :

$$
\frac{f^{\prime}(\phi) d \phi}{\lambda\left(\phi-\phi_{+}\right)+\Delta_{+}-f(\phi)}=c d \xi .
$$

Since $\phi_{+}$satisfies $f\left(\phi_{+}\right)=\Delta_{+}$, write

$$
f(\phi)-\Delta_{+}=g(\phi)\left(\phi-\phi_{+}\right) .
$$

Equation (5.3) can be integrated to yield

$$
c\left(\xi-\xi_{0}\right)=\ln \left|\frac{\phi_{0}-\phi_{+}}{\phi-\phi_{+}}\right|+\ln \left|\frac{\lambda-g\left(\phi_{0}\right)}{\lambda-g(\phi)}\right|+\lambda \int_{\phi_{0}}^{\phi} \frac{d \phi^{\prime}}{\left(\phi^{\prime}-\phi_{+}\right)\left(\lambda-g\left(\phi^{\prime}\right)\right)},
$$

where

$$
\phi_{0}=\lim _{\xi \rightarrow \xi_{0}+} \phi(\xi)
$$

and the final quadrature requires specification of $g(\phi)$.

As $\xi \rightarrow+\infty$ we require $\phi \rightarrow \phi_{+}$. Anticipating that

$$
\phi-\phi_{+}=\mathrm{O}\left(e^{-\kappa \xi}\right) \text {, }
$$

we find from (5.5) that

$$
\kappa=c\left\{1-\lambda / g\left(\phi_{+}\right)\right\}
$$

Since from (5.4)

$$
g\left(\phi_{+}\right)=f^{\prime}\left(\phi_{+}\right),
$$

which by assumption (2.12) is negative, $\kappa$ is positive and (5.5) defines an acceptable solution.

Turning to $\xi<\xi_{0}$, formally the solution appears to be given by (5.5) with $\phi_{+}$ replaced by $\phi_{-}$and $g$ now defined by $f(\phi)-\Delta_{-}=\left(\phi-\phi_{-}\right) g(\phi)$. However, the resulting solution behaves as $\phi \sim \phi_{-}+\mathrm{O}\left(\exp \left(-\kappa^{\prime} \xi\right)\right)$ as $\xi \rightarrow-\infty$ with $\kappa^{\prime}>0$, which is unacceptable. Hence for $\xi<\xi_{0}$ we take $\phi \equiv \phi_{-}$and $u \equiv-\Delta_{-}$. Our final solution is then

$$
\begin{aligned}
& \phi(\xi)=\left\{\begin{array}{lll}
\phi_{-} & \text {if } & \xi<\xi_{0}, \\
\Phi_{0}\left(c\left(\xi-\xi_{0}\right)\right) & \text { if } & \xi>\xi_{0}
\end{array}\right. \\
& u(\xi)= \begin{cases}-\Delta_{-} & \text {if } \xi<\xi_{0}, \\
-f\left(\Phi_{0}\left(c\left(\xi-\xi_{0}\right)\right)\right) & \text { if } \quad \xi>\xi_{0},\end{cases}
\end{aligned}
$$


where $\Phi_{0}(\zeta)$ is defined by inverting (5.5) for $\phi$ as a function of $\zeta=c\left(\xi-\xi_{0}\right)$. The unknown value $\phi_{0}$ appearing in (5.5) can be determined if we maintain continuity of $u$ across $\xi_{0}$. Hence $\phi_{0}$ is the solution of

$$
f\left(\phi_{0}\right)=\Delta_{-}
$$

with $\phi_{0}$ near +1 .

By construction $u$ is continuous. However, $\phi(\xi)$ is clearly discontinuous. Direct calculation establishes that $\boldsymbol{u}^{\prime}(\xi)$ is also discontinuous with

$$
u^{\prime}\left(\xi_{0}+\right)-u^{\prime}\left(\xi_{0}-\right)=-c \lambda\left(\phi_{0}-\phi_{-}\right),
$$

where we have made use of (4.10).

If we specialize to $\Delta_{-}=0$, which corresponds to the 'solid' being at the melting temperature, and for which $\phi_{0}=-\phi_{-}=1,(5.13)$ has a direct and familiar interpretation. Returning to physical variables (5.13) reads

$$
\left(\frac{d T}{d x}\right)_{x_{0}-}-\left(\frac{d T}{d x}\right)_{x_{0}+}=c\left(\frac{L}{c_{s} D}\right)
$$

which is precisely (1.1) specialized to a planar interface. The recovery of the usual Stefan boundary condition should not be surprising, since the conventional derivation of (1.1) relies on the conservation of energy across the (sharp) front. In a phase field model this conservation is automatically ensured by the thermodynamic foundation.

The velocity $c$ is clearly not determined, solutions existing for all $c>0$. In this sense, the solution we have constructed is qualitatively identical to that found for a steady-state planar front in the conventional Stefan problem (see, for example, [27]). In that case, $u^{\prime}$ is discontinuous, as it has to be to satisfy (1.1), while $u$ decays as $e^{-c \xi}$ as $\xi \rightarrow \infty$ with $c$ indeterminate. How this indeterminancy is removed in a phase field model with non-zero $\epsilon$ is the subject of the next few sections.

Before turning to this analysis, we specialize for future reference (5.11) to $\phi^{4}$-theory. In this case, $g(\phi)$ is a quadratic:

$$
g(\phi)=\frac{1}{2}\left(1-\phi^{2}-\phi \phi_{+}-\phi_{+}^{2}\right)
$$

so that the final integral in (5.5) is elementary. If

$$
\mu_{ \pm}=-\frac{1}{2}\left(\phi_{+} \mp \sqrt{4-3 \phi_{+}^{2}-8 \lambda}\right)
$$

denote the zeros of $\lambda-g(\phi)$ then the function $\Phi_{0}(\zeta)$ is given by solving

$$
\begin{aligned}
\zeta= & \frac{1-3 \phi_{+}^{2}}{2 \lambda-1+3 \phi_{+}^{2}} \ln \left|\frac{\Phi_{0}-\phi_{+}}{\phi_{0}-\phi_{+}}\right|-\ln \left|\frac{2 \lambda-1+\Phi_{0}^{2}+\Phi_{0} \phi_{+}+\phi_{+}^{2}}{2 \lambda-1+\phi_{0}^{2}+\phi_{0} \phi_{+}+\phi_{+}^{2}}\right| \\
& +\frac{2 \lambda}{\sqrt{4-3 \phi_{+}^{2}-8 \lambda}}\left\{\frac{1}{\left(\mu_{+}-\phi_{+}\right)} \ln \left|\frac{\Phi_{0}-\mu_{+}}{\phi_{0}-\mu_{+}}\right|-\frac{1}{\left(\mu_{-}-\phi_{+}\right)} \ln \left|\frac{\Phi_{0}-\mu_{-}}{\phi_{0}-\mu_{-}}\right|\right\}
\end{aligned}
$$


for $\Phi_{0}$.

\section{Matched asymptotic expansions: $\epsilon \rightarrow 0, \tau=O(\epsilon)$}

We now investigate the effect of small but finite $\epsilon$ and $\tau$ on the steady-state solutions derived in the preceding section. Presumably in this case the discontinuities in $\phi$ and $u^{\prime}$ are smoothed. We might also expect that the non-uniqueness of the velocity is removed with a specific value being selected. A mathematical complication arises from the fact that two small parameters exist. In this section we resolve this difficulty by assuming that

$$
\tau=\tau_{1} \epsilon \text {. }
$$

The basic steady-state phase field equations now read:

$$
\begin{aligned}
\epsilon^{2} \phi^{\prime \prime}+c \tau_{1} \epsilon \phi^{\prime}+f(\phi)+u & =0 \\
u^{\prime \prime}+c\left(u^{\prime}+\lambda \phi^{\prime}\right) & =0
\end{aligned}
$$

and can be analysed for small $\epsilon$ by application of matched asymptotic expansions. The method is standard [23].

We assume that $\phi$ and $u$ possess outer expansions:

$$
\begin{aligned}
& \phi=\phi_{\text {outer }}(\xi ; \epsilon)=\phi_{0}^{o}(\xi)+\epsilon \phi_{1}^{o}(\xi)+\cdots, \\
& u=u_{\text {outer }}(\xi ; \epsilon)=u_{0}^{o}(\xi)+\epsilon u_{1}^{o}(\xi)+\cdots
\end{aligned}
$$

Substituting these expansions in (6.2) and (6.3) and equating successive powers of $\epsilon$ yields

$$
\begin{aligned}
& f\left(\phi_{0}^{o}\right)+u_{0}^{o}=0, \\
& u_{0}^{o \prime}+c\left(u_{0}^{o \prime}+\lambda \phi_{0}^{o \prime}\right)=0, \\
& c \tau_{1} \phi_{0}^{o \prime}+\phi_{1}^{o} f^{\prime}\left(\phi_{0}^{o}\right)+u_{1}^{o}=0, \\
& u_{1}^{o \prime \prime}+c\left(u_{1}^{o \prime}+\lambda \phi_{1}^{o \prime}\right)=0,
\end{aligned}
$$

etc. The velocity $c=c(\epsilon)$ should also be expanded as

$$
c=c_{0}+c_{1} \epsilon+\cdots
$$

to consistently derive the outer equations. However, since $c$ will not be constrained by the outer solutions it is convenient to omit this step at this stage. We shall assume 
that $c=\mathrm{O}(1)$ as $\epsilon \rightarrow 0$ and that the boundary data is indepedent of $\epsilon$. If the boundary conditions are satisfied at zeroth order, that is,

$$
\phi_{0}^{o} \rightarrow \phi_{ \pm}, \quad u_{0}^{o} \rightarrow-\Delta_{ \pm} \text {as } \xi \rightarrow \pm \infty,
$$

then the higher order functions satisfy

$$
\phi_{k}^{o} \rightarrow 0, \quad u_{k}^{o} \rightarrow 0 \text { as } \xi \rightarrow \pm \infty
$$

Finally, the no flux boundary conditions at infinity imply that

$$
\phi_{k}^{o \prime} \rightarrow 0 \quad \text { and } \quad u_{k}^{o \prime} \rightarrow 0 \text { as } \xi \rightarrow \pm \infty
$$

for all $k \geq 0$.

The zeroth order equations are precisely the problem treated in the preceding section. Hence $\phi_{0}^{o}$ and $u_{0}^{o}$ are determined by (5.10) and (5.11) with $c$ (strictly $c_{0}=$ $\left.\lim _{\epsilon \rightarrow 0} c(\epsilon)\right)$ undetermined.

The first-order corrections follow from (6.8) and (6.9). For $\xi<\xi_{0}$, since $\phi_{0}^{o}=\phi_{-}$, we have

$$
\phi_{1}^{o}=-u_{1}^{o} / f^{\prime}\left(\phi_{-}\right)
$$

so that (6.9) reduces to

$$
u_{1}^{o \prime \prime}+c u_{1}^{o \prime}\left(1-\frac{\lambda}{f^{\prime}\left(\phi_{-}\right)}\right)=0,
$$

which is to be solved subject to the boundary conditions $u_{1}^{o} \rightarrow 0, u_{1}^{o \prime} \rightarrow 0$ as $\xi \rightarrow-\infty$. The only acceptable solution is

$$
u_{1}^{o}=0 \text {, }
$$

which implies, via (6.14), that

$$
\phi_{1}^{o}=0 \text {. }
$$

For $\xi>\xi_{0}$, the analysis is slightly more complicated since $\phi_{0}^{o}$ now depends on $\xi$. From (6.8) we have

$$
\phi_{1}^{o}(\xi)=-\frac{u_{1}^{o}(\xi)+c \tau_{1} \phi_{0}^{o \prime}(\xi)}{f^{\prime}\left(\phi_{0}^{o}(\xi)\right)}
$$

Integrating (6.9) and applying the boundary condition as $\xi \rightarrow \infty$ yields

$$
u_{1}^{o \prime}+c\left(u_{1}^{o}+\lambda \phi_{1}^{o}\right)=0,
$$

which, on substituting (6.18) for $\phi_{1}^{o}$, reduces to

$$
u_{1}^{o \prime}+c \kappa(\xi) u_{1}^{o}=\frac{c^{2} \lambda \tau_{1} \phi_{0}^{o \prime}(\xi)}{f^{\prime}\left(\phi_{0}^{o}(\xi)\right)}
$$


where

$$
\kappa(\xi)=\left(1-\frac{\lambda}{f^{\prime}\left(\phi_{0}^{o}(\xi)\right)}\right) .
$$

Equation (6.20) is to be solved subject to the condition

$$
u_{1}^{o}\left(\xi_{0}\right)=0 \text {. }
$$

We also require that $u_{1}^{o} \rightarrow 0$ as $\xi \rightarrow \infty$. However, since $\kappa(\xi) \rightarrow\left(1-\lambda / f\left(\phi_{+}\right)\right)>0$ as $\xi \rightarrow \infty$, this is automatic. Integrating (6.20) yields

$$
u_{1}^{o}(\xi)=c^{2} \lambda \tau_{1} \int_{\xi_{0}}^{\xi} \frac{\phi_{0}^{o \prime}\left(\xi^{\prime}\right)}{f^{\prime}\left(\phi_{0}^{o}\left(\xi^{\prime}\right)\right)} \exp \left[-c \int_{\xi^{\prime}}^{\xi} \kappa(s) d s\right] d \xi^{\prime}
$$

The integral over $\kappa$ can be evaluated in closed form by changing the variable of integration to $w=\phi_{0}^{o}(s)$. Hence from (6.21) and (5.3) we obtain

$$
c \int_{\xi^{\prime}}^{\xi} \kappa(s) d s=\ln \left|\frac{f\left(\phi_{0}^{o}\left(\xi^{\prime}\right)\right)-\Delta_{+}-\lambda\left(\phi_{0}^{o}\left(\xi^{\prime}\right)-\phi_{+}\right)}{f\left(\phi_{0}^{o}(\xi)\right)-\Delta_{+}-\lambda\left(\phi_{0}^{o}(\xi)-\phi_{+}\right)}\right| .
$$

Substituting this result in (6.23) we find that the resulting expression for $u_{1}^{0}$ simplifies if we regard $u_{1}^{0}$ (for $\xi \geq 0$ ) as a function of $\phi_{0}^{o}(\xi)$. Explicitly, write

$$
u_{1}^{0}(\xi)=c^{2} \lambda \tau_{1} \mathcal{U}\left(\phi_{0}^{o}(\xi)\right)
$$

where, from (6.23),

$$
\mathcal{U}(\phi)=\int_{\phi_{0}}^{\phi} \frac{d w}{f^{\prime}(w)}\left\{\frac{f(\phi)-\Delta_{+}-\lambda\left(\phi-\phi_{+}\right)}{f(w)-\Delta_{+}-\lambda\left(w-\phi_{+}\right)}\right\},
$$

with $\phi_{0}=\phi_{0}^{o}\left(\xi_{0}\right)$. For $\phi^{4}$-theory, this final quadrature is also tractable; the integrand being a rational function. We shall make use of these analytical results in Section 8 .

The outer expansions are now to be augmented by appropriately matched inner expansions obtained by introducing the stretched variable

$$
z=\left(\xi-\xi_{0}\right) / \epsilon .
$$

In terms of $z$ the basic phase field equations read

$$
\begin{aligned}
\phi^{\prime \prime}+c \tau_{1} \phi^{\prime}+f(\phi)+u & =0, \\
u^{\prime \prime}+c \epsilon\left(u^{\prime}+\lambda \phi^{\prime}\right) & =0,
\end{aligned}
$$

where ' now denotes differentiation with respect to $z$. 
Substituting the inner expansions

$$
\begin{aligned}
& \phi=\phi_{\text {inner }}(z ; \epsilon)=\phi_{0}^{i}(z)+\epsilon \phi_{1}^{i}(z)+\cdots, \\
& u=u_{\text {inner }}(\xi ; \epsilon)=u_{0}^{i}(z)+\epsilon u_{1}^{i}(z)+\cdots,
\end{aligned}
$$

for $\phi$ and $u$, together with the expansion (6.10) for $c$, in (6.28) and (6.29) yield, on equating successive powers of $\epsilon$,

$$
\begin{aligned}
& u_{0}^{i \prime \prime}=0, \\
& \phi_{0}^{i \prime \prime}+c_{0} \tau_{1} \phi_{0}^{i^{\prime}}+f\left(\phi_{0}^{i}\right)+u_{0}^{i}=0, \\
& u_{1}^{i^{\prime \prime}}+c_{0}\left(u_{0}^{i^{\prime}}+\lambda \phi_{0}^{i^{\prime}}\right)=0, \\
& \phi_{1}^{i^{\prime \prime}}+c_{1} \tau_{1} \phi_{0}^{i^{\prime}}+c_{0} \tau_{1} \phi_{1}^{i^{\prime}}+f^{\prime}\left(\phi_{0}^{i}\right) \phi_{1}^{i}+u_{1}^{i}=0,
\end{aligned}
$$

etc.

The necessary data to ensure unique solutions to these equations follow by appropriately matching to the outer solution $[23,37]$. Hence from $(6.32)$ we find that

$$
u_{o}^{i}=-\Delta_{-},
$$

while $\phi_{0}^{i}$ satisfies

$$
\phi_{0}^{i \prime \prime}+c_{0} \tau_{1} \phi_{0}^{i^{\prime}}+f\left(\phi_{0}^{i}\right)-\Delta_{-}=0
$$

subject to the boundary conditions

$$
\phi_{0}^{i} \rightarrow \phi_{ \pm}^{0} \quad \text { as } \quad z \rightarrow \pm \infty
$$

where $\phi_{ \pm}^{0}$ are roots of $f(\phi)=\Delta_{-}$with $\phi_{-}^{0} \equiv \phi_{-}$near -1 and $\phi_{+}^{0}$ near +1 .

Equations of the form of (6.37) have been discussed by Hagan [16, 17]. While existence for arbitrary $f$ does not appear to have been established, Hagan [17] did prove that if a solution existed for a particular value, say $c^{*}(\Delta)$, of the 'eigenvalue' $c_{0} \tau_{1}$ then this value was unique and the solution was also unique up to translation. For $\phi^{4}$-theory, the solution can be constructed explicitly. Direct substitution shows that

$$
\phi_{0}^{i}(z)=\frac{1}{2}\left(\phi_{+}^{0}+\phi_{-}^{0}\right)+\frac{1}{2}\left(\phi_{+}^{0}-\phi_{-}^{0}\right) \tanh \alpha_{0} z,
$$

is a solution of (6.37) provided

$$
\alpha_{0}=\frac{1}{4}\left(\phi_{+}^{0}-\phi_{-}^{0}\right)=-\frac{1}{2} \sin \theta\left(\Delta_{-}\right)
$$

and

$$
c_{0} \tau_{1}=c^{*}(\Delta)=-\frac{3}{2}\left(\phi_{+}^{0}+\phi_{-}^{0}\right)=\sqrt{3} \cos \theta\left(\Delta_{-}\right),
$$


where $\theta\left(\Delta_{-}\right)$is given by (4.13). By Hagan's theorem [17, Theorem 5] this solution is unique apart from translations. For small $\Delta_{-}$:

$$
\begin{aligned}
\alpha_{0} & =\left(1-3 \Delta_{-}^{2}+O\left(\Delta_{-}^{3}\right)\right) / 2, \\
c_{0} \tau_{1} & =3 \Delta_{-}+O\left(\Delta_{-}^{2}\right) .
\end{aligned}
$$

With the zeroth-order functions $\phi_{0}^{i}$ and $u_{0}^{i}$ evaluated and $c_{0}$ known, the first-order corrections follow in principle from (6.34) and (6.35). Even within $\phi^{4}$-theory the necessary calculations cannot be carried out analytically. However, it is possible to evaluate $c_{1}$.

Integrating (6.34) once gives

$$
u_{1}^{i^{\prime}}=-c_{0} \lambda \phi_{0}^{i}+A .
$$

The constant $A$ can be determined by matching this result in the limit $z \rightarrow-\infty$ with the outer expansion in the limit $\xi \rightarrow \xi_{0}-$. Since to $O(\epsilon), u_{\text {outer }}=0$ this gives $A=c_{0} \lambda \phi_{-}$. Hence integrating (6.44) and similarily matching to determine the constant of integration, we obtain

$$
u_{1}^{i}=-c_{0} \lambda \int_{-\infty}^{z}\left[\phi_{0}^{i}\left(z^{\prime}\right)-\phi_{-}\right] d z^{\prime}
$$

Turning now to the calculation of $\phi_{1}^{i}$ we write (6.34) as

$$
\mathcal{L} \phi_{1}^{i}=-u_{1}^{i}-c_{1} \tau_{1} \phi_{0}^{i^{\prime}} \equiv r(z),
$$

where

$$
\mathcal{L}=\frac{d^{2}}{d z^{2}}+c_{0} \tau_{1} \frac{d}{d z}+f^{\prime}\left(\phi_{0}^{i}(z)\right) .
$$

The following facts pertaining to $\mathcal{L}$ are easily established.

(i) With respect to the usual $L_{2}$ inner product, the adjoint of $\mathcal{L}$ is

$$
\mathcal{L}^{\dagger}=\frac{d^{2}}{d z^{2}}-c_{0} \tau_{1} \frac{d}{d z}+f^{\prime}\left(\phi_{0}^{i}(z)\right)
$$

(ii) Differentiating (6.37) with respect to $z$ implies that $\mathcal{L} \phi_{0}^{i^{\prime}}=0$.

(iii) By direct calculation

$$
\mathcal{L}^{\dagger}\left\{e^{c_{0} \tau_{1} z} \phi_{0}^{i^{\prime}}\right\}=e^{c_{0} \tau_{1} z} \mathcal{L} \phi_{0}^{i^{\prime}}=0
$$

(iv) Hence

$$
w(z)=e^{c_{0} \tau_{1} z} \phi_{0}^{i^{\prime}}(z)
$$

is a null vector of $\mathcal{L}^{\dagger}$. 
Consequently, a necessary condition for $\phi_{1}^{i}$ to exist is that $w$ is orthogonal to the inhomogeneous term in (6.46), that is

$$
\langle w, r\rangle=\int_{-\infty}^{\infty} w(z) r(z) d z=0 .
$$

Since all functions are known this condition determines $c_{1}$, namely

$$
\tau_{1} c_{1}=-\frac{\int_{-\infty}^{\infty} u_{1}^{i}(z) w(z) d z}{\int_{-\infty}^{\infty} \phi_{0}^{i^{\prime}}(z) w(z) d z},
$$

where $u_{1}^{i}$ is given by (6.45).

Specializing to $\phi^{4}$-theory we have

$$
u_{1}^{i}=-2 c_{0} \lambda\left(\alpha_{0} z+\ln 2 \cosh \alpha_{0} z\right),
$$

which yields

$$
c_{1} \tau_{1}=c_{0} \lambda \alpha_{0}^{-2} K_{0}\left(c_{0} \tau_{1} / 2 \alpha_{0}\right) / J\left(c_{0} \tau_{1} / 2 \alpha_{0}\right)
$$

where

$$
K_{0}(p)=\int_{-\infty}^{\infty}(s+\ln 2 \cosh s) e^{2 p s} \operatorname{sech}^{2} s d s
$$

and

$$
J(p)=\int_{-\infty}^{\infty} e^{2 p s} \operatorname{sech}^{4} s d s .
$$

The substitution $v=1 /\left(1+e^{2 s}\right)$ transforms both integrals to standard integrals that can be evaluated [15, page $294, \# 3.251$ and page $538, \# 4.253$ ] in terms of gamma functions. Hence

$$
\begin{aligned}
J(p) & =\frac{4}{3} \Gamma(2-p) \Gamma(2+p)=\frac{4}{3} \pi p\left(1-p^{2}\right) \operatorname{cosec} \pi p, \\
K_{0}(p) & =2 \Gamma(1-p) \Gamma(1+p)\left[1-C_{E}-\psi(1-p)\right] \\
& =2 \pi p\left[1-C_{E}-\psi(1-p)\right] \operatorname{cosec} \pi p,
\end{aligned}
$$

where $C_{E}=0.577216 \ldots$ is Euler's constant and $\psi(z)=d(\ln \Gamma(z)) / d z$ is the logarithmic derivative of the gamma function. Combining these results gives

$$
c_{1} \tau_{1}=\frac{3 c_{0} \lambda}{2 \alpha_{0}^{2}}\left(1-p^{2}\right)^{-1}\left[1-C_{E}-\psi(1-p)\right], \quad p=c_{0} \tau_{1} / 2 \alpha_{0},
$$

for the first-order correction for the velocity. As $z \rightarrow 1$,

$$
\psi(z)=-C_{E}-\left(\pi^{2} / 6\right)(1-z)+O\left((1-z)^{2}\right),
$$

which together with the expansions (6.42) and (6.43) for $\alpha_{0}$ and $c_{0}$, implies that

$$
c_{1}\left(\Delta_{-}\right)=18 \lambda \tau_{1}^{-2} \Delta_{-}+\mathrm{O}\left(\Delta_{-}^{2}\right) \quad \text { as } \quad \Delta_{-} \rightarrow 0 .
$$




\section{Arbitrary $\epsilon$ and $\tau$-interpretation as a dynamical system}

In the preceding section we were able to demonstrate the existence and selection of a unique steady-state planar front for the phase field equations in the limit $\epsilon \rightarrow 0$ with $\tau=\mathrm{O}(\epsilon)$. While physically we expect $\epsilon$ and $\tau$ to be small, we know of no compelling argument that suggests that they should be proportional. Indeed, in most of the existing literature on phase field models, it is usually assumed that $\tau=O\left(\epsilon^{2}\right)$, again without any compelling argument. In this section we reinterpret ${ }^{7}$ the basic steady-state phase field equations as a first-order dynamical system with the aim of exploring the extent to which we can establish the existence of steady-state fronts without an assumption of an explicit relation between $\epsilon$ and $\tau$.

We assume, for non-zero $\epsilon$ and $\tau$, that any acceptable solution must be at least $\mathcal{C}^{2}$, if not, as one might anticipate physically, $\mathcal{C}^{\infty}$. Hence we may replace (4.3) by the first-order equation (4.8). Changing the independent variable to

$$
s=\xi / \epsilon,
$$

we can write the basic equations as

$$
\begin{aligned}
\phi^{\prime \prime}+\delta \hat{\tau} \phi^{\prime}+f(\phi)+u & =0, \\
u^{\prime}+\delta\left(u+\Delta_{-}+\lambda\left(\phi-\phi_{-}\right)\right) & =0,
\end{aligned}
$$

where

$$
\hat{\tau}=\tau / \epsilon^{2}
$$

and

$$
\delta=c \epsilon
$$

These equations are subject to the usual boundary conditions, namely

$$
u \rightarrow-\Delta_{ \pm}, \quad \phi \rightarrow \phi_{ \pm} \quad \text { as } \quad s \rightarrow \pm \infty
$$

with, in particular,

$$
\Delta_{+}-\Delta_{-}=\lambda\left(\phi_{+}-\phi_{-}\right) .
$$

Equations (7.2) and (7.3) can be intrepreted in a number of different ways. If, as in the preceding section, we assume that $\tau$ and $\epsilon$ are related but now as $\tau=\hat{\tau} \epsilon^{2}$, then (7.2) and (7.3) constitute the inner equations replacing (6.28) and (6.29). The problem is that $\epsilon$ no longer appears in the equations and an inner expansion is not possible. Alternatively, we can simply regard $\hat{\tau}$ as a material parameter. In either case the question of the existence of steady-state fronts and the selection of their velocity, if they exist, reduces to the question of the existence of solutions to the boundary 


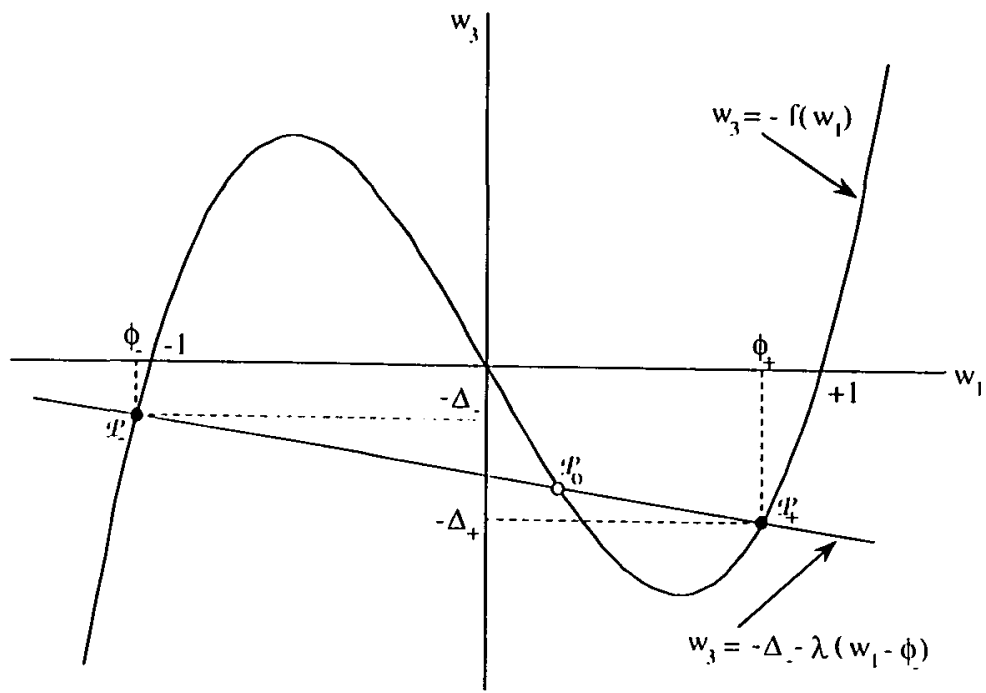

FIGURE 1. Schematic representation of the fixed point structure in the $w_{2}=0$ plane of the system (7.9). Travelling wave solutions correspond to orbits joining the fixed points $\mathcal{P}_{-}$and $\mathcal{P}_{+}$.

value problem defined by (7.2) and (7.3) and the boundary conditions (7.6) with the velocity entering through the eigenvalue $\delta$.

It is convenient to rewrite (7.2) and (7.3) as a third-order dynamical system by defining phase-space variables

$$
w_{1}=\phi, \quad w_{2}=\phi^{\prime}, \quad w_{3}=u .
$$

In terms of the $w_{i}$ 's (7.2) and (7.3) become

$$
\begin{aligned}
& w_{1}^{\prime}=w_{2}, \\
& w_{2}^{\prime}=-w_{3}-f\left(w_{1}\right)-\delta \hat{\tau} w_{2}, \\
& w_{3}^{\prime}=-\delta\left(w_{3}+\Delta_{-}+\lambda\left(w_{1}-\phi_{-}\right)\right) .
\end{aligned}
$$

By inspection this system exhibits fixed points at values of $w_{1}, w_{2}$ and $w_{3}$ that satisfy

$$
w_{2}=0, \quad w_{3}=-f\left(w_{1}\right)=-\Delta_{-}-\lambda\left(w_{1}-\phi_{-}\right) .
$$

If the function $f$ has the usual properties, this fixed point condition can be intrepreted graphically as in Figure 1 . In the physically relevant regime of parameters, three fixed points exist, which we denote

$$
\mathcal{P}_{\sigma}=w^{\sigma}=\left(w_{1}^{\sigma}, 0, w_{3}^{\sigma}\right)=\left(\phi_{\sigma}, 0,-\Delta_{\sigma}\right), \quad \sigma= \pm, 0 .
$$

${ }^{7}$ Similar ideas have been discussed recently by Wilder [39] for the special case of $\phi^{4}$-theory. 
As the notation suggests, the points $\mathcal{P}_{ \pm}$correspond to the boundary conditions satisfied by the required front solutions. Hence the existence of a steady-state planar front corresponds [39] to the existence of a heteroclinic orbit joining $\mathcal{P}_{-}$and $\mathcal{P}_{+}$. We have not been able to establish this existence in general. However, as we report in detail in the next section, numerical results for $\phi^{4}$-theory suggest that such an orbit exists only for a unique value of $\delta$. This conclusion is supported by the behaviour of the solution in the vicinity of the fixed points.

This behaviour follows in the standard way by linearizing (7.9) about the appropriate fixed point. Write

$$
w=w^{\sigma}+\omega
$$

then to first order in $\omega,(7.9)$ reduces to

$$
\omega^{\prime}=A_{\sigma} \omega,
$$

where

$$
A_{\sigma}=\left(\begin{array}{ccc}
0 & 1 & 0 \\
\gamma_{\sigma} & -\delta \hat{\tau} & -1 \\
-\delta \lambda & 0 & -\delta
\end{array}\right)
$$

with $\gamma_{\sigma}=-f^{\prime}\left(\phi_{\sigma}\right)$.

If the eigenvalues of $A_{\sigma}$ are denoted $q_{i}^{\sigma}, i=1,2,3$, we observe that

$$
\begin{aligned}
\operatorname{Tr} A_{\sigma} & =\sum q_{i}^{\sigma}=-\delta(1+\hat{\tau})<0, \\
\operatorname{det} A_{\sigma} & =\prod q_{i}^{\sigma}=\delta\left(\gamma_{\sigma}+\lambda\right) .
\end{aligned}
$$

By assumption (2.12) $\gamma_{ \pm}>0$. Hence $\operatorname{det} A_{ \pm}$is positive and, at the fixed points $\mathcal{P}_{ \pm}$, two possibilities exist:

(i) All $q_{i}^{ \pm}$are real with $q_{1}^{ \pm}>0>q_{2}^{ \pm} \geq q_{3}^{ \pm}$.

(ii) $q_{1}^{ \pm}$is real while $q_{2}^{ \pm}$and $q_{3}^{ \pm}$are complex conjugate pairs with $\operatorname{Re} q_{2}^{ \pm}=\operatorname{Re} q_{3}^{ \pm}<$ 0 .

Hence as $s \rightarrow-\infty$,

$$
\left(\begin{array}{c}
\phi \\
\phi^{\prime} \\
u
\end{array}\right) \sim\left(\begin{array}{c}
\phi_{-} \\
0 \\
-\Delta_{-}
\end{array}\right)+C_{-} e^{q_{1}^{-} s}\left(\begin{array}{c}
1 \\
q_{1}^{-} \\
-q_{1}^{-} \lambda /\left(q_{1}^{-}+\delta\right)
\end{array}\right),
$$

where the second term arises from the right eigenvector associated with the eigenvalue $q_{1}^{-}$and $C_{-}$is a constant. Since the system is autonomous $C_{-}$can be set to, say, unity. The question now is: Does this solution, that is completely specified as $s \rightarrow-\infty$, approach $\left(\phi_{+}, 0-\Delta_{+}\right)^{\dagger}$ as $s \rightarrow+\infty$ ? Since the attractive subspace of the fixed point $\mathcal{P}_{+}$is only two-dimensional this seems unlikely unless the only free parameter $\delta$ is appropriately tuned. This heuristic argument suggests that a hetroclinic orbit joining $\mathcal{P}_{-}$and $\mathcal{P}_{+}$exists only at most for particular values of $\delta$. Our numerical results for $\phi^{4}$-theory suggest that there is at most one allowable value of $\delta$. 


\section{Numerical results: $\phi^{4}$-theory}

We now specialize to $\phi^{4}$-theory and explore the existence of steady-state solutions to the phase field equations numerically. Specifically we seek solutions to (4.2) and (4.3), which now read

$$
\begin{aligned}
\epsilon^{2} \phi^{\prime \prime}+\tau c \phi^{\prime}+\phi\left(1-\phi^{2}\right) / 2+u & =0 \\
u^{\prime \prime}+c\left(u^{\prime}+\lambda \phi^{\prime}\right) & =0
\end{aligned}
$$

subject to

$$
u \rightarrow-\Delta_{ \pm}, \quad \phi \rightarrow \phi_{ \pm} \quad \text { as } \quad \xi \rightarrow \pm \infty,
$$

where the boundary data $\Delta_{+}$and $\phi_{ \pm}$are given in terms of $\Delta_{-}$by (4.14)-(4.16). (Note that we have returned to $\xi$ as the independent variable.)

From the analysis of the previous section, expressed in $\xi$ and $c$, we know that if a solution exists then

$$
\phi(\xi) \sim \begin{cases}\phi_{-}+C_{-} e^{\kappa_{-} \xi}, & \text { as } \xi \rightarrow-\infty \\ \phi_{+}+C_{+} e^{-\kappa_{+} \xi}, & \text { as } \xi \rightarrow+\infty\end{cases}
$$

and

$$
u(\xi) \sim \begin{cases}-\Delta_{-}-c \lambda C_{-} e^{\kappa_{-} \xi} /\left(c+\kappa_{-}\right), & \text {as } \xi \rightarrow-\infty \\ -\Delta_{+}+c \lambda C_{+} e^{-\kappa_{+} \xi} /\left(c-\kappa_{+}\right), & \text {as } \xi \rightarrow+\infty\end{cases}
$$

Here $\kappa_{-}=q_{1}^{-} / \epsilon$, where $q_{1}^{-}$is the positive eigenvalue of $A_{-}$and $\kappa_{+}=-q_{1}^{+} / \epsilon$, where $q_{1}^{+}$is the negative eigenvalue of $A_{+}$of smallest magnitude. ${ }^{8}$ The constant $C_{-}$may be chosen arbitrarily by appropriate choice of the translational degree of freedom.

As posed, (8.1) and (8.2) constitute a relatively straightforward boundary value problem. The only computational difficulties arise from the infinite interval and the stiffness of (8.1) for small $\epsilon$. The interval of integration can be replaced by a finite interval $(-L, L)$ with effective boundary conditions at $\xi= \pm L$ defined from (8.4) and (8.5). The resulting boundary value problem has two parameters $c$ and the constant $C_{+}$in (8.4) and (8.5) that can be adjusted to find a solution. This adjustment was achieved by imposing continuity of $\phi^{\prime}$ at $\xi= \pm L$ and using Newton iteration on the discretised system. The free constant $C_{-}$was chosen to ensure that $\phi^{\prime}(-L)$ was not neglible. The Newton iteration tended to become unstable as $\epsilon \rightarrow 0$. While this instablity could be overcome to some extent with a continuation method, the singular nature of the system ultimately halted convergence for $\epsilon$ less than about $3 \times 10^{-2}$.

Figure 2 shows the numerical solution found by this method for $\epsilon=0.034$ and the indicated values of the material parameters, where we have set $\tau=\tau_{1} \epsilon$ to allow comparison with the results of Section 6 . The corresponding velocity is $c=0.7583$.

\footnotetext{
${ }^{8}$ For the parameter regimes of relevance $A_{+}$has two real negative eigenvalues.
} 


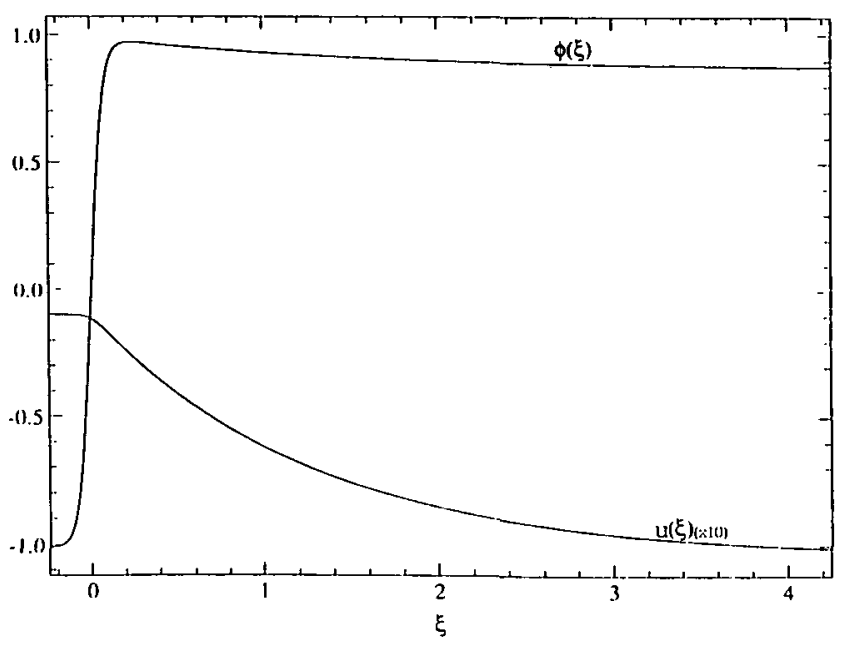

FIGURE 2. Graphs of the numerical solutions of the steady-state solutions to the phase field equations for $\Delta_{-}=0.01, \lambda=0.05, \tau=\tau_{1} \epsilon, \tau_{1}=0.05, \epsilon=0.034$. The velocity is $c=0.7583$. Note that values of $u$ have been multiplied by a factor of 10 .

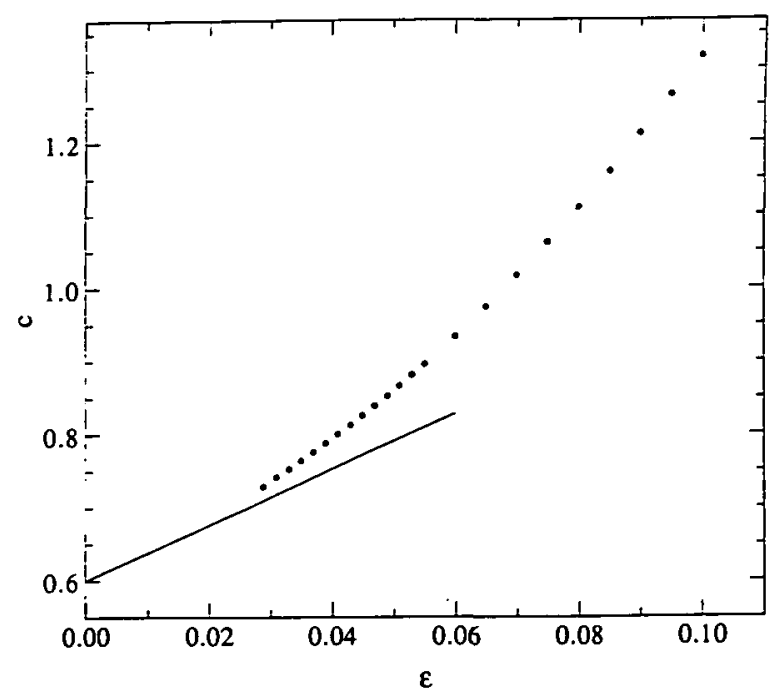

FIGURE 3. Variation of steady-state velocity, $c$, as a function of $\epsilon$ for $\Delta_{-}=0.01, \lambda=0.05, \tau=\tau_{1} \epsilon$, $\tau_{1}=0.05$. The straight line is the asymptotic result, $c(\epsilon)=c_{0}+c_{1} \epsilon$, derived in Section 6 . 


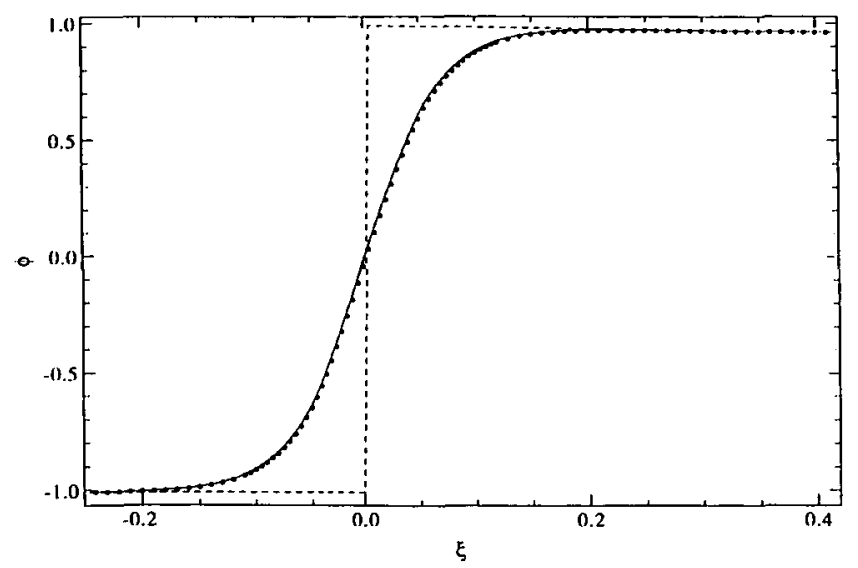

FIGURE 4. Comparison of the numerical calculation of $\phi(\xi)$ (solid circles) with the outer 'Stefan-like' solution given by (8.6) (- . - - ) and the uniform expansion (8.11) and (8.13) (-). Material parameters have the same values as in Figure 2.

The variation of the velocity $c$ with $\epsilon$ is illustrated in Figure 3, where we also show the asymptotic result, $c(\epsilon)=c_{0}+c_{1} \epsilon$, that follows from (6.41) and (6.59). For the indicated parameter values, $c_{0}=0.600240 \ldots$ and $c_{1}=3.787822 \ldots$. While the numerical results are not in the strict asymptotic region, convergence to the predicted behaviour is clear.

Figures 4 and 5 present a more detailed comparison of the numerical results for $\phi$ and $u$ with the analytical results of Sections 5 and 6 . In particular, we compare with the "Stefan-like" (or zeroth-order outer) solution of Section 5 and a uniform asymptotic approximation constructed from the inner and outer expansions derived in Section 6 . The former is defined by

$$
\phi_{o}^{0}(\xi)= \begin{cases}-1 & \text { for } \xi<0 \\ \Phi_{0}(c \xi) & \text { for } \xi \geq 0\end{cases}
$$

and

$$
u_{o}^{0}(\xi)= \begin{cases}-\Delta_{-} & \text {for } \xi<0 \\ -\frac{1}{2} \Phi_{0}(c \xi)\left(1-\Phi_{0}^{2}(c \xi)\right) & \text { for } \xi \geq 0\end{cases}
$$

where $\Phi_{0}$ is determined ${ }^{9}$ by (5.17) and $c$ is replaced by $c_{0}+c_{1} \epsilon$. The discontinuities in $\phi$ and $u^{\prime}$ are evident.

These discontinuities are smoothed in the uniform approximation. This was constructed in the standard way (see, for example, [37]) by adding the outer and inner

${ }^{9}$ We have set the arbitrary constant $\xi_{0}$ to zero. 


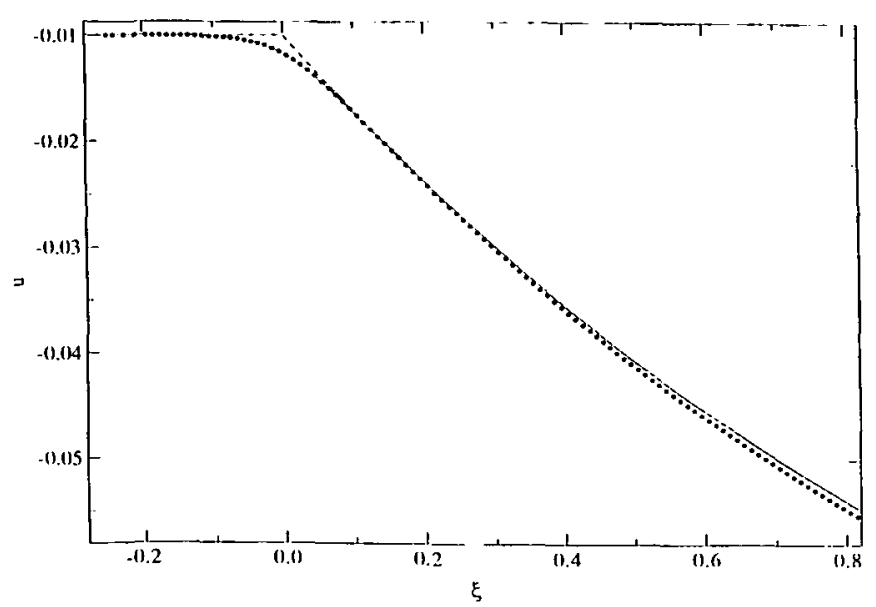

FIGURE 5. Comparison of the numerical calculation of $u(\xi)$ (solid circles) with the outer 'Stefan-like' solution given by (8.7) (- - - -) and the uniform expansion (8.10) and (8.12) (-). Material parameters have the same values as in Figure 2.

solutions and subtracting the part that they have in common. In our case this prescription leads to the approximation

$$
u_{\text {unif }}(\xi)=u_{0}^{o}(\xi)+u_{0}^{i}(\xi / \epsilon)+\epsilon\left[u_{1}^{o}(\xi)+u_{1}^{i}(\xi / \epsilon)\right]-u_{\text {com }}(\xi),
$$

where $u_{\text {com }}(\xi)$ can be obtained by expanding $u_{\text {inner }}(\xi / \epsilon)$ to $\mathrm{O}(\epsilon)$ at fixed $\xi$. Similarly

$$
\phi_{\text {unif }}(\xi)=\phi_{0}^{o}(\xi)+\phi_{0}^{i}(\xi / \epsilon)+\epsilon \phi_{1}^{o}(\xi)-\phi_{\text {com }}(\xi),
$$

where $\phi_{\mathrm{com}}(\xi)$ is obtained by expanding $\phi_{\text {inner }}(\xi / \epsilon)$ to $\mathrm{O}(1)$ in $\epsilon$ at fixed $\xi$. Note that, in light of the available results from Section 6 , we have included only the zeroth-order term in the inner expansion of $\phi$.

Specializing to $\phi^{4}$-theory we have, for $\xi<0$,

$$
\begin{aligned}
& u_{\text {unif }}(\xi)=-\Delta_{-}-2 c_{0} \lambda\left[\alpha_{0} \xi+\epsilon \ln \left(2 \cosh \left(\alpha_{0} \xi / \epsilon\right)\right)\right], \\
& \phi_{\text {unif }}(\xi)=\frac{1}{2}\left(\phi_{+}^{0}+\phi_{-}^{0}\right)+\frac{1}{2}\left(\phi_{+}^{0}-\phi_{-}^{0}\right) \tanh \left(\alpha_{0} \xi / \epsilon\right),
\end{aligned}
$$

and for $\xi \geq 0$,

$$
\begin{aligned}
u_{\text {unif }}(\xi)= & -\frac{1}{2} \Phi_{0}(c \xi)\left(1-\Phi_{0}^{2}(c \xi)\right)+c^{2} \lambda \tau_{1} \epsilon \mathcal{U}\left(\Phi_{0}(c \xi)\right) \\
& +2 c_{0} \lambda\left[\alpha_{0} \xi-\epsilon \ln \left(2 \cosh \left(\alpha_{0} \xi / \epsilon\right)\right)\right] \\
\phi_{\text {unif }}(\xi)= & \Phi_{0}(c \xi)+\frac{1}{2}\left(\phi_{+}^{0}-\phi_{-}^{0}\right)\left(\tanh \left(\alpha_{0} \xi / \epsilon\right)-1\right) \\
& -2 c^{2} \tau_{1} \epsilon\left\{\frac{\lambda \mathcal{U}\left(\Phi_{0}(c \xi)\right)-\Phi_{0}^{\prime}(c \xi)}{1-3 \Phi_{0}^{2}(c \xi)}\right\} .
\end{aligned}
$$


In these expressions $\Phi_{0}$ is given by (5.17), $\mathcal{U}$ by integratinq (6.26) and we again replace $c$ by $c_{0}+c_{1} \epsilon$. Inspection of Figures 4 and 5 reveals that these approximants are in excellent agreement with the numerical results. The deviation evident in Figure 5 can be accounted for by the small discrepancy $(\approx 4 \%)$ between $c_{0}+c_{1} \epsilon$ and the true value of $c$.

We now turn to the case of arbitrary $\tau$ and $\epsilon$ and consider the solution of (7.2) and (7.3). Obviously, these can be solved in a similar way as a boundary value problem. However, it is more instructive to approach the solution by an alternative method $^{10}$ which exploits the formulation as a dynamical system directly.

We firstly extend the result (7.17) to a complete asymptotic series valid as $s \rightarrow-\infty$. Integrating (7.3) formally gives

$$
u(s)=-\Delta_{-}-\lambda \delta \int_{0}^{\infty} e^{-\delta t}\left(\phi(s-t)-\phi_{-}\right) d t,
$$

which allows (7.2) and (7.3) to be combined into a single integro-differential equation:

$$
\phi^{\prime \prime}+\delta \hat{\tau} \phi^{\prime}+\frac{1}{2} \phi\left(1-\phi^{2}\right)=\Delta_{-}+\lambda \delta \int_{0}^{\infty} e^{-\delta t}\left(\phi(s-t)-\phi_{-}\right) d t .
$$

Defining

$$
v(s)=\phi(s)-\phi_{-},
$$

we write (8.15) as

$$
\mathcal{K}_{-} v=v^{2}\left(3 \phi_{-}+v\right) / 2,
$$

where $\mathcal{K}_{-}$is a linear operator defined by

$$
\mathcal{K}_{-} v=\frac{d^{2} v}{d s^{2}}+\delta \hat{\tau} \frac{d v}{d s}-\gamma_{-} v-\lambda \delta \int_{0}^{\infty} e^{-\delta t} v(s-t) d t
$$

with

$$
\gamma_{-}=-f^{\prime}\left(\phi_{-}\right)=-\left(1-3 \phi_{-}^{2}\right) / 2
$$

We observe that

$$
\mathcal{K}_{-} e^{p s}=W_{-}(p) e^{p s}
$$

where

$$
W_{-}(p)=p^{2}+\delta \hat{\tau} p-\gamma_{-}-\frac{\lambda \delta}{p+\delta} .
$$

This expression can be written as $W_{-}(p)=\operatorname{det}\left(A_{-}-p I\right) /(p+\delta)$, where $A_{-}$is the matrix defined in (7.14). Since this matrix has, by the arguments in Section 7, only

${ }^{10} \mathrm{~A}$ similar method that was suggested some time ago by Ablowitz and Zeppetella [1]. 
one positive eigenvalue we immediately conclude that $W_{-}(p)$ has only one positive zero which we denote $p_{0}$.

To leading order as $s \rightarrow-\infty$, the quadratic terms in (8.17) can be neglected and we recover the asymptotic behaviour

$$
v(s) \sim C_{1} e^{p_{0} s}, \quad \text { as } \quad s \rightarrow-\infty .
$$

We now extend this result by assuming that the solution can be represented as a Liapunov-Poincaré expansion [28] of the form

$$
v(s)=\sum_{m=1}^{\infty} C_{m} e^{m p_{0} s} .
$$

Substituting this expansion in (8.15) we find that the coefficients $C_{m}$ can be determined by the recurrence relations:

$$
C_{2}=\frac{3 \phi_{-} C_{1}^{2}}{2 W_{-}\left(2 p_{0}\right)}
$$

and

$$
C_{m}=\frac{1}{2 W_{-}\left(m p_{0}\right)}\left\{3 \phi_{-} \sum_{n=1}^{m-1} C_{n} C_{m-n}+\sum_{n=2}^{m-1} \sum_{k=1}^{n-1} C_{k} C_{n-k} C_{m-n}\right\}, \quad m \geq 3 .
$$

We observe that $C_{1}$ is arbitrary and can be removed by defining $C_{m}=C_{1}^{m} \hat{C}_{m}$, where the $\hat{C}_{m}$ 's satisfy (8.24) and (8.25) with $\hat{C}_{1}=1$. This scaling simply reflects the translational invariance of the system.

Hence as $s \rightarrow-\infty$, we have the representations:

$$
\begin{aligned}
& \phi(s)=\phi_{-}+\sum_{m=1}^{\infty} C_{m} e^{m p_{0} s}, \\
& \phi^{\prime}(s)=p_{0} \sum_{m=1}^{\infty} m C_{m} e^{m p_{0} s}, \\
& u(s)=-\Delta_{-}-\lambda \delta \sum_{m=1}^{\infty} \frac{C_{m}}{\delta+m p_{0}} e^{m p_{0} s},
\end{aligned}
$$

where the last expression for $u$ follows from (8.14). Similar representations can be derived in the limit $s \rightarrow \infty$. However, these are more complicated because of the two roots of the characteristic equation that contribute to the asymptotic behaviour.

Instead we use the representations (8.26), (8.27) and (8.28) with a finite number of terms (we used a maximum of 50) to evaluate $\phi, \phi^{\prime}$ and $u$ up to some maximum value of $s$, say $s=s_{0}$, for a specified value of $\delta$. At $s_{0}$ these values are then used as initial 

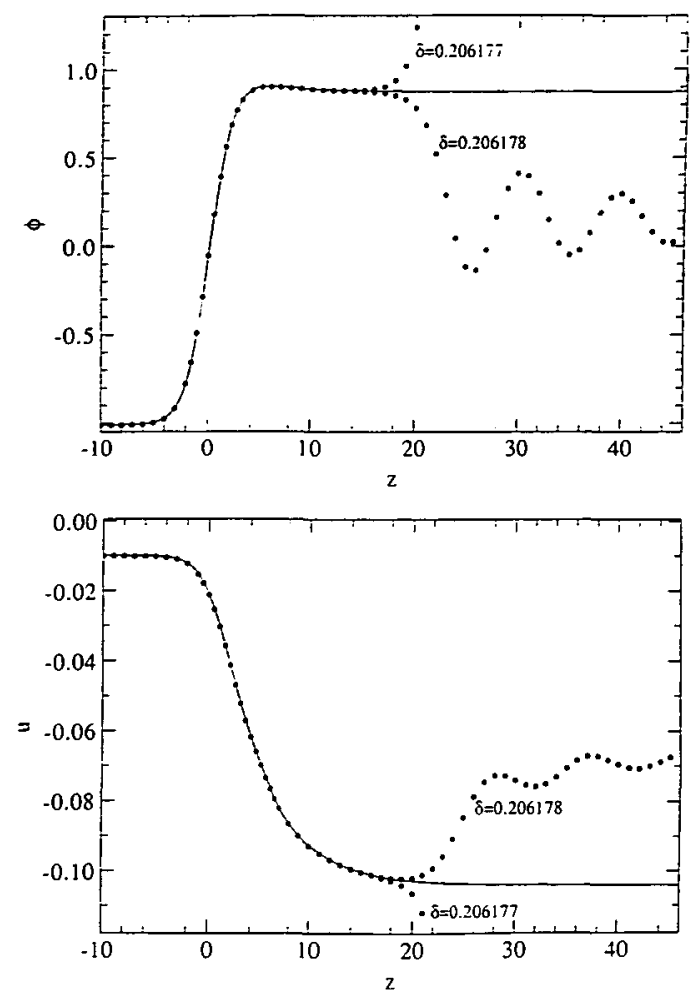

FIGURE 6. Typical behaviour as a function of $\delta$ of the solutions to the initial value problem described in the text. Shown are plots of (a) $\phi$ and (b) $u$. The solid line is the solution to the corresponding boundary value problem in which the correct boundary conditions are imposed at both limits. The material parameters are $\lambda=0.05, \hat{\tau}=0.4$ and $\Delta_{-}=0.01$.

values to integrate the dynamical system defined in (7.9) numerically by a standard intitial value solver. ${ }^{11}$ Depending on $\delta$ the ensuing trajectory in phase space either diverges to large $\phi$ (and $\phi^{\prime}$ ) or apparently approaches a finite limit that is indepenedent of $\delta$ (but dependent on parameters such as $\Delta_{-}, \lambda$, etc.). Typical results for $\phi$ and $u$ are shown in Figures 6 and 7; the latter being a blow-up of the corresponding region of Figure 6a. The switch between the two types of behaviour appears to occur at a unique value $\delta_{c}=\delta_{c}\left(\Delta_{-}\right)$of $\delta$ : orbits with $\delta<\delta_{c}$ diverging while those with $\delta>\delta_{c}$ tending to the finite limit.

This limit is not, however, the fixed point $\mathcal{P}_{+}$. Instead the orbit is attracted to the third fixed point $\mathcal{P}_{0}$ identified in Section 7 , which for the relevant parameter values is an attractive node with two complex eigenvalues whose imaginary parts match

\footnotetext{
"We used the NAG routine D02EBF which is a variable-order, variable-step implementation of the backward differentaition formulae [18].
} 


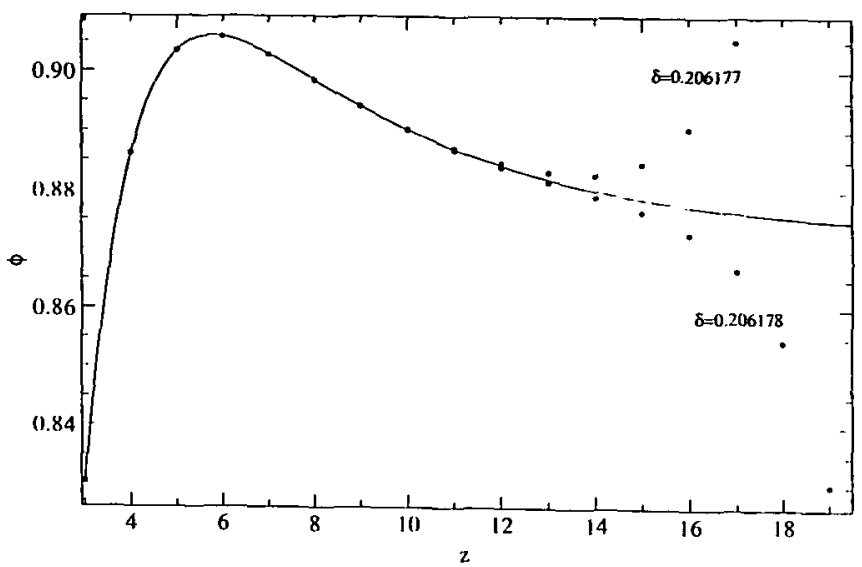

FIGURE 7. Blow-up of the corresponding region of Figure 6a.

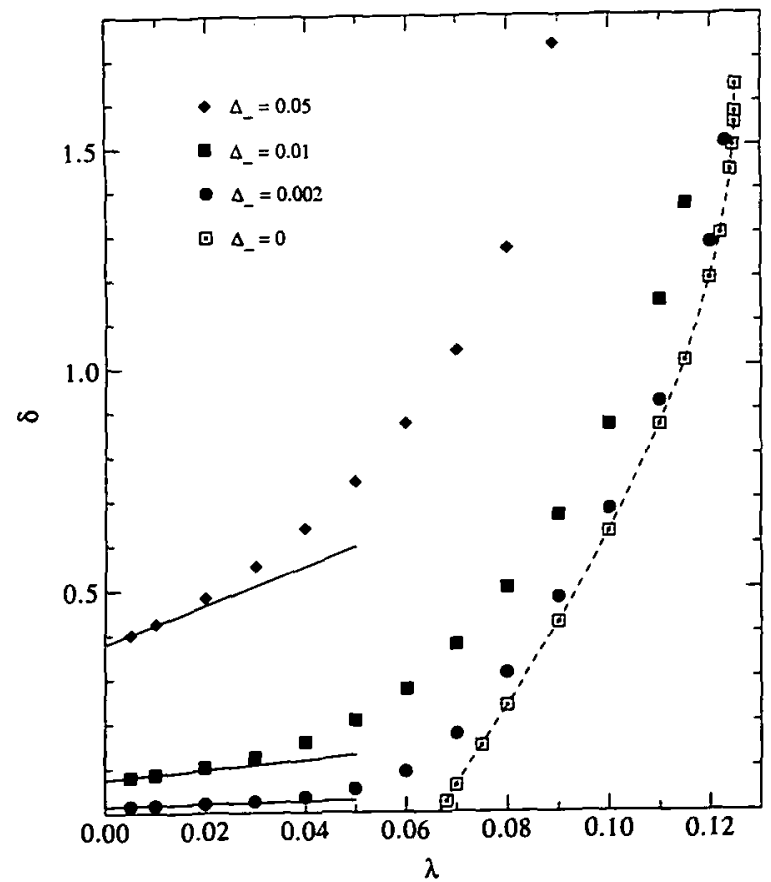

FIGURE 8. Variation of the selected value of $\delta$ as a function of $\lambda$ for the indicated values of $\Delta_{-}$. (The material parameter $\hat{\tau}=0.4$.) The solid lines represent exact asymptotic results (to $O(\lambda)$ ) derived in Section 9. The broken line through the data points for $\Delta_{-}=0$ is drawn only as a guide. 
precisely the observed oscillations.

Thus for neither $\delta>\delta_{c}$ nor $\delta<\delta_{c}$ do orbits apparently approach the fixed point $\mathcal{P}_{+}$and hence define acceptable solutions to the boundary value problem (8.15). Such a solution does appear to arise for $\delta=\delta_{c}$ as indicated in Figure 6 , where we superimposed the solution obtained by solving the boundary value problem directly. This comparison leads us to conjecture: Steady-state travelling planar fronts of the phase field equations exist only for the unique velocity $c(\epsilon)=\delta_{c} / \epsilon$. This front is morever unique except for translation. While we are unable to rigorously prove this conjecture for arbitrary values of the physical parameters, it can be confirmed analytically in the limit of small $\lambda$ as we show in the next section. We also observe from (8.1) that if $u<0$ and $\phi>1$ then $\phi^{\prime}+\delta \hat{\tau} \phi$ is a monotone increasing function of $s$. Hence any trajectory that enters the region $\left\{w_{3}<0\right.$ and $\left.w_{1}>1\right\}$ of phase space must diverge.

The critical value $\delta_{c}$ depends on the material parameters. The dependence on $\lambda$ is shown in Figure 8 for several values of $\Delta_{-}$. The striking feature is the behaviour of $\delta_{c}$ for $\Delta_{-}=0$, for which a critical value $\lambda_{c}$ of $\lambda$ appears to exist such that

$$
\lim _{\Delta_{-} \rightarrow 0} \delta_{c}\left(\Delta_{-}\right)= \begin{cases}0 & \text { if } \lambda \leq \lambda_{c} \\ \delta_{c}(0)>0 & \text { if } \lambda>\lambda_{c} .\end{cases}
$$

Numerically, $\lambda_{c}=\hat{\tau} / 6$, a value that we will confirm analytically in Section 10 .

\section{Expansion in $\lambda$}

The numerical results presented in the preceding section, together with the heuristic argument devloped in Section 7, constitute clear evidence for the existence of a unique steady-state velocity for travelling planar fronts in phase field models. In this section we confirm this conclusion analytically to $O(\lambda)$ in an expansion about the limit $\lambda=0$.

A convenient starting point for the derivation of the required expansion is the integro-differential equation (8.15) which, on integrating by parts and generalizing to an arbitrary function $f(\phi)$, can be rewritten as

$$
\phi^{\prime \prime}+\delta \hat{\tau} \phi^{\prime}+f(\phi)=\Delta_{-}+\lambda \int_{-\infty}^{s}\left[1-e^{-\delta(s-t)}\right] \phi^{\prime}(t) d t
$$

where

$$
\phi(s) \rightarrow \phi_{ \pm} \quad \text { as } \quad s \rightarrow \pm \infty
$$

the boundary data being subject to the usual conditions. In terms of $\phi$,

$$
u(s)=-\Delta_{-}-\lambda \int_{-\infty}^{s}\left[1-e^{-\delta(s-t)}\right] \phi^{\prime}(t) d t .
$$


We now assume that

$$
\phi(s ; \lambda)=\phi_{0}(s)+\lambda \phi_{1}(s)+\mathrm{O}\left(\lambda^{2}\right),
$$

and

$$
\delta(\lambda)=\delta_{0}+\lambda \delta_{1}+\mathrm{O}\left(\lambda^{2}\right),
$$

with $\delta_{0} \neq 0$. Substituting these expansions in (9.1) and equating successive powers of $\lambda$ yields:

$$
\begin{gathered}
\phi_{0}^{\prime \prime}+\delta_{0} \hat{\tau} \phi_{0}^{\prime}+f\left(\phi_{0}\right)-\Delta_{-}=0, \\
\phi_{1}^{\prime \prime}+\delta_{0} \hat{\tau} \phi_{1}^{\prime}+f^{\prime}\left(\phi_{0}(s)\right) \phi_{1}=-\delta_{1} \hat{\tau} \phi_{0}^{\prime}+\int_{-\infty}^{s}\left[1-e^{-\delta_{0}(s-t)}\right] \phi_{0}^{\prime}(t) d t,
\end{gathered}
$$

etc.

Similarily expanding the boundary data gives

$$
\Delta_{+}=\Delta_{-}+\lambda\left(\phi_{+}^{0}-\phi_{-}\right)+\mathrm{O}\left(\lambda^{2}\right)
$$

and

$$
\phi_{-}=\phi_{-}^{0}, \quad \phi_{+}=\phi_{+}^{0}+\lambda\left(\phi_{+}^{0}-\phi_{-}\right) / f^{\prime}\left(\phi_{+}^{0}\right)+\mathrm{O}\left(\lambda^{2}\right),
$$

where, as before,

$$
f\left(\phi_{ \pm}^{0}\right)=\Delta_{-}
$$

with $\phi_{-}^{0} \sim-1$ and $\phi_{+}^{0} \sim+1$. Thus we require

$$
\begin{gathered}
\phi_{0}(s) \rightarrow \phi_{ \pm}^{0} \quad \text { as } \quad s \rightarrow \pm \infty, \\
\phi_{1}(s) \rightarrow \begin{cases}0 & \text { as } s \rightarrow-\infty, \\
\left(\phi_{+}^{0}-\phi_{-}^{0}\right) / f^{\prime}\left(\phi_{+}^{0}\right) & \text { as } s \rightarrow+\infty,\end{cases}
\end{gathered}
$$

with corresponding boundary conditions on the higher order functions that can be derived by extending the expansions in (9.8) and (9.9).

The equation for $\phi_{0}$ is identical to (6.37) that arose in the inner expansion discussed in Section 6. Thus the conclusions reached there are immediately applicable: $\phi_{0}(s)$ exists if an only if $\delta_{0} \hat{\tau}$ takes the unique value $c^{*}$. In particular, transcribing the results of Section 6, we have within $\phi^{4}$-theory:

$$
\phi_{0}(s)=\frac{1}{2}\left(\phi_{+}^{0}+\phi_{-}^{0}\right)+\frac{1}{2}\left(\phi_{+}^{0}-\phi_{-}^{0}\right) \tanh \alpha_{0} s,
$$

where

$$
\alpha_{0}=\frac{1}{4}\left(\phi_{+}^{0}-\phi_{-}^{0}\right)=-\frac{1}{2} \sin \theta\left(\Delta_{-}\right)
$$

and

$$
\delta_{0} \hat{\tau}=-\frac{3}{2}\left(\phi_{+}^{0}+\phi_{-}^{0}\right)=\sqrt{3} \cos \theta\left(\Delta_{-}\right) \text {, }
$$


with $\theta(\Delta)$ defined in (4.13), namely

$$
\theta(\Delta)=\frac{1}{3} \cos ^{-1}(-3 \sqrt{3} \Delta)-\frac{2 \pi}{3} .
$$

Likewise the first-order correction $\phi_{1}(s)$ satisfies an inhomogeneous linear equation

$$
\mathcal{L} \phi_{1}=R(s),
$$

where $R(s)$ denotes the right-hand side of (9.7). The linear operator $\mathcal{L}$ is the same linear operator (recall (6.47)) that arose in the inner expansion of Section 6. Consequently, for $\phi_{1}$ to exist we require $R(s)$ to be orthogonal to the null vector of $\mathcal{L}^{\dagger}$. As in Section 6, the only unknown free parameter in this condition is $\delta_{1}$. Hence we conclude that

$$
\hat{\tau} \delta_{1}=\frac{\int_{-\infty}^{\infty} e^{\delta_{0} \hat{t} s} \phi_{0}^{\prime}(s) \int_{-\infty}^{s}\left[1-e^{-\delta_{0}(s-t)}\right] \phi_{0}^{\prime}(t) d t d s}{\int_{-\infty}^{\infty} e^{\delta_{0} \hat{\tau} s}\left[\phi_{0}^{\prime}(s)\right]^{2} d s}
$$

For $\phi^{4}$-theory, this expression is conveniently rewritten as

$$
\hat{\tau} \delta_{1}=\frac{\delta_{0}}{\alpha_{0}^{2}} \frac{K\left(\delta_{0} \hat{\tau} / 2 \alpha_{0}, \delta_{0} / 2 \alpha_{0}\right)}{J\left(\delta_{0} \hat{\tau} / 2 \alpha_{0}\right)},
$$

where the functions $K$ and $J$ are defined by

$$
K(p, q)=\int_{-\infty}^{\infty} e^{2 p t} \operatorname{sech}^{2} t d t \int_{-\infty}^{t} e^{-2 q\left(t-t^{\prime}\right)}\left(1+\tanh t^{\prime}\right) d t^{\prime}
$$

and

$$
J(p)=\int_{-\infty}^{\infty} e^{2 p t} \operatorname{sech}^{4} t d t
$$

which is the same integral evaluated in Section 6, recall (6.56).

Except for the case $p=q$, it does not appear possible to express the function $K(p, q)$ as simply. The substitutions $u^{\prime}=e^{2 t^{\prime}}, u=e^{2 t}$ transform (9.20) to a form that can be integrated [15, page $284, \# 3.194]$ in terms of generalized hypergeometric functions. Explicitly we find

$$
\begin{aligned}
K(p, q) & =\frac{2}{1+q} \int_{0}^{\infty} \frac{u^{1+p}}{(1+u)^{2}}{ }_{2} F_{1}(1,1+q ; 2+q ;-u) d u \\
& =\frac{1+p}{1+q} \frac{\pi p}{\sin \pi p}{ }_{3} F_{2}(1,1,2+p ; 2+q, 3 ; 1),
\end{aligned}
$$

where ${ }_{\mu} F_{\nu}$ denotes a generalized hypergeometric function with ${ }_{2} F_{1}$ the standard function. Hence

$$
\hat{\tau} \delta_{1}=\frac{3 \delta_{0}}{4 \alpha_{0}^{2}} \frac{{ }_{3} F_{2}\left(1,1,2+\hat{\tau} q_{0} ; 2+q_{0}, 3 ; 1\right)}{\left(1-\hat{\tau} q_{0}\right)\left(1+q_{0}\right)}, \quad q_{0}=\frac{\delta_{0}}{2 \alpha_{0}} .
$$




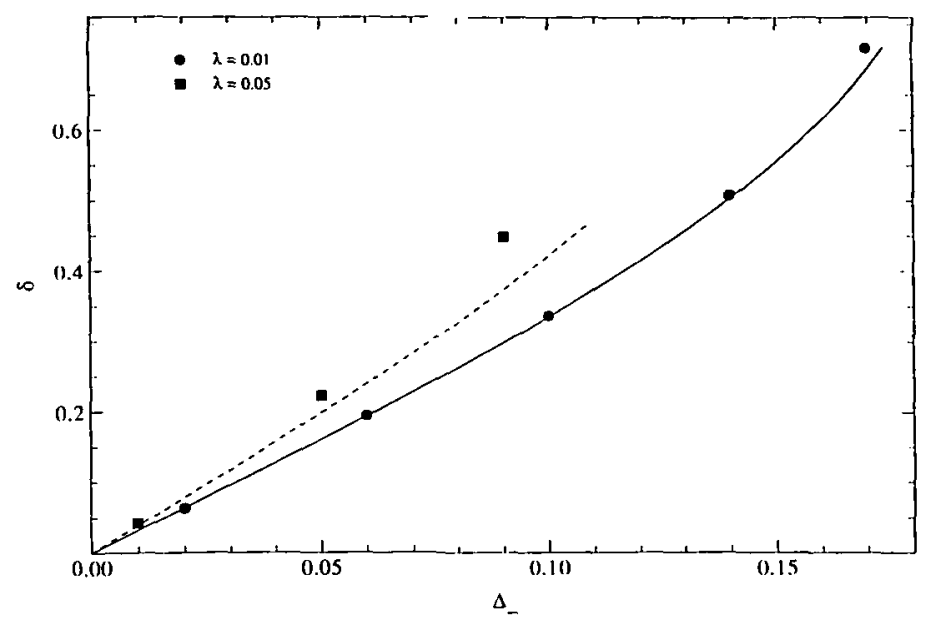

FIGURE 9. Comparison of the asymptotic result (9.25) for $\hat{\boldsymbol{\tau}}=1$ as a function of $\Delta_{-}$. The points are exact numerical values of $\delta$ while the lines are plots of $(9.25)$ truncated at $O(\lambda)$ and evaluated for $\lambda=0.01$ (solid line) and $\lambda=0.05$ (dashed line), respectively.

For the special case $p=q$, corresponding to $\hat{\tau}=1,{ }_{3} F_{2}$ reduces to an ordinary hypergeometric function of unit argument, which yields

$$
K(p, p)=2 \pi p \operatorname{cosec} \pi p
$$

Hence for $\hat{\tau}=1$, we have

$$
\delta\left(\lambda, \Delta_{-}\right)=\sqrt{3} \cos \theta\left\{1-\frac{6 \lambda}{1+2 \cos 2 \theta}+O\left(\lambda^{2}\right)\right\}
$$

where $\theta=\theta\left(\Delta_{-}\right)$is given by (9.16). Figure 9 compares this asymptotic result, truncated at $O(\lambda)$, with some exact numerical values of $\delta$. The agreement is excellent for even relatively large values of $\lambda$; recall that $\lambda$ is bounded for fixed $\Delta_{-}$, see (4.17).

For other values of $\hat{\tau}$, it is necessary to evaluate ${ }_{3} F_{2}$ and hence $\delta_{1}$ numerically. However, this is relatively easy if the series definition of ${ }_{3} F_{2}$ is appropriately accelerated, see Appendix A. The results are the straight lines shown on Figure 8 of the previous section. Agreement with the numerical values of $\delta$ for sufficiently small $\lambda$ is excellent.

There is obviously a close similarity between the results found in this section through expansions in $\lambda$ and those of Section 6 derived by matched asymptotics in $\epsilon$ with $\tau=\tau_{1} \epsilon$. We can, in fact, recover the latter from (9.15) and (9.19) if we set

$$
\hat{\imath}=\tau / \epsilon^{2}=\tau_{1} / \epsilon, \quad \delta=c \epsilon
$$


and consider the limit $\epsilon \rightarrow 0$ with $\delta \hat{\tau}=c \tau_{1}=\mathrm{O}(1)$. Making these substitutions in (9.15) and (9.19) we obtain

$$
\tau_{1} c(\epsilon)=\sqrt{3} \cos \theta+\frac{\lambda \epsilon}{\alpha_{0} \tau_{1}} \Lambda(-\sqrt{3} \cot \theta ; \epsilon)+\mathrm{O}\left(\lambda^{2}\right),
$$

where $\alpha_{0}=-\frac{1}{2} \sin \theta$, with $\theta$ still determined in terms of $\Delta_{-}$by (9.16), and

$$
\Lambda(p ; \epsilon)=\frac{p K\left(p, p \epsilon / \tau_{1}\right)}{J(p)} .
$$

Expanding (9.20) for small $q$ gives

$$
K(p, q)=K_{0}(p)+\mathrm{O}(q),
$$

where $K_{0}(p)$ is defined in (6.55). Thus writing

$$
\tau_{1} c(\epsilon)=c_{0} \tau_{1}+c_{1} \tau_{1} \epsilon+\mathrm{O}\left(\epsilon^{2}\right),
$$

we obtain

$$
c_{0} \tau_{1}=\sqrt{3} \cos \theta+\mathrm{O}\left(\lambda^{2}\right)
$$

and

$$
c_{1} \tau_{1}=\frac{\lambda c_{0} \tau_{1}}{2 \alpha_{0}^{2}} \frac{K_{0}\left(c_{0} \tau_{1} / 2 \alpha_{0}\right)}{J\left(c_{0} \tau_{1} / 2 \alpha_{0}\right)}+\mathrm{O}\left(\lambda^{2}\right) .
$$

These results are precisely the results of Section 6, namely (6.41) and (6.54), with apparently the terms of order $\lambda^{2}$ identically zero.

$$
\text { 10. The limit } \lambda \rightarrow 0, \hat{\tau}=O(\lambda), \Delta_{-}=O(\lambda)
$$

The results of the preceding section confirm the numerical results shown in Figure 7 of Section 8 for small $\lambda$. However, the nature of the expansions developed in Section 9 preclude investigation of the appearance of steady-state solutions for $\Delta_{-}=0$ and $\lambda$ sufficiently large. This aspect can be explored if we consider the limit $\lambda \rightarrow 0$ with

$$
\hat{\tau}=\hat{\tau}_{1} \lambda \quad \text { and } \quad \Delta_{-}=d_{1} \lambda .
$$

Proceeding as in the previous section, we assume that a solution to the integrodifferential equation (9.7) exists of the form

$$
\phi(s)=\phi_{0}(s)+\lambda \phi_{1}(s)+\mathrm{O}\left(\lambda^{2}\right),
$$

with

$$
\delta=\delta_{0}+\lambda \delta_{1}+\mathrm{O}\left(\lambda^{2}\right), \quad \delta_{0} \neq 0
$$


Substituting in (9.7) yields the sequence of equations:

$$
\begin{gathered}
\phi_{0}^{\prime \prime}+f\left(\phi_{0}\right)=0, \\
\phi_{1}^{\prime \prime}+f^{\prime}\left(\phi_{0}(s)\right) \phi_{1}=d_{1}-\delta_{0} \hat{\tau}_{1} \phi_{0}^{\prime}(s)+\int_{-\infty}^{s}\left[1-e^{-\delta_{0}(s-t)}\right] \phi_{0}^{\prime}(t) d t,
\end{gathered}
$$

etc. Expanding the boundary data on $\phi$ implies that these equations are to be solved subject to the boundary conditions

$$
\phi_{0}(s) \rightarrow \pm 1, \quad \phi_{0}^{\prime}(s) \rightarrow 0, \quad \text { as } \quad s \rightarrow \pm \infty
$$

and

$$
\phi_{1}(s) \rightarrow \phi_{1}^{ \pm}, \quad \phi_{1}^{\prime}(s) \rightarrow 0 \quad \text { as } \quad s \rightarrow \pm \infty,
$$

where $\phi_{1}^{-}=d_{1} / f^{\prime}(-1)$ and $\phi_{1}^{+}=\left(2+d_{1}\right) / f^{\prime}(+1)$.

The argument is now familar. The existence of a unique solution, modulo translation, to (10.4) satisfing the boundary conditions (10.6) is well-known, see for example, [14]. Moreover, $\phi_{0}$ is such that

$$
\left|\phi_{0} \mp 1\right| \leq C e^{-\kappa|s|}, \quad\left|\phi_{0}^{\prime}\right| \leq C^{\prime} e^{-\kappa|s|} \quad \text { as } \quad s \rightarrow \pm \infty,
$$

where $C, C^{\prime}$ and $\kappa$ are positive constants [14]. In our case, since $f(\phi)=-\Psi^{\prime}(\phi)$ with $\Psi(\phi)>\Psi( \pm 1)=0$ for $\phi \neq \pm 1$, we can assert that $\phi_{0}^{\prime}(s)>0$ and

$$
s-s_{0}=\int_{0}^{\phi_{0}} \frac{d \phi}{\sqrt{2 \Psi(\phi)}},
$$

where $s_{0}$ is an arbitrary constant such that $\phi_{0}\left(s_{0}\right)=0$. For $\phi^{4}$-theory this integral reproduces the kink solution

$$
\phi_{0}(s)=\tanh (s / 2)
$$

where, without loss of generality, we have set $s_{0}=0$.

With $\phi_{0}$ determined, $\phi_{1}$ again satisfies a linear inhomogenous equation

$$
\mathcal{L}_{0} \phi_{1}=\bar{R}(s),
$$

where $\bar{R}(s)$ denotes the right-hand side of (10.5) and

$$
\mathcal{L}_{0}=\frac{d^{2}}{d s^{2}}+f^{\prime}\left(\phi_{0}(s)\right)
$$

is a self-adjoint operator with null vector $\phi_{0}^{\prime}(s)$. Hence a necessary condition for $\phi_{1}$ to exist is that $\delta_{0}$ satisfies the solvability criterion

$$
\int_{-\infty}^{\infty} \bar{R}(s) \phi_{0}^{\prime}(s) d s=0
$$




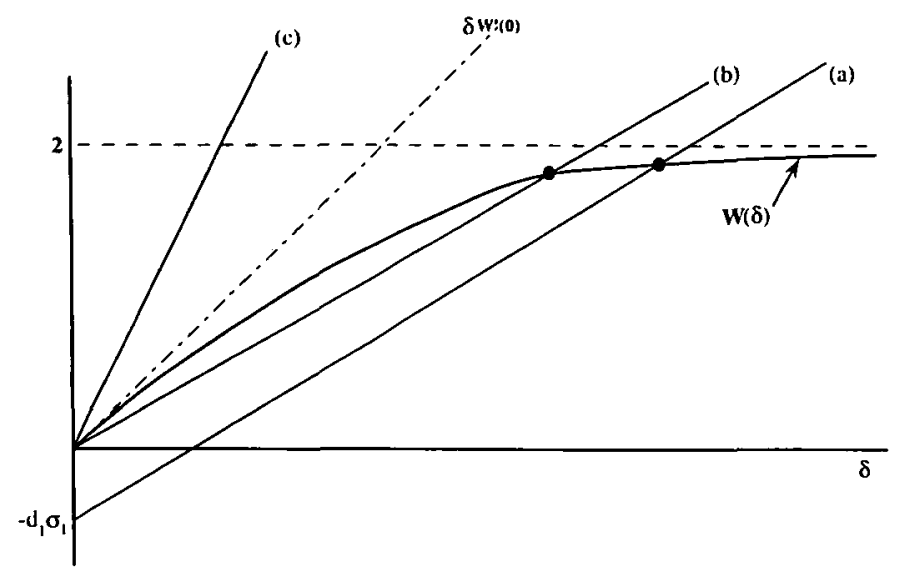

FIGURE 10. Qualitative behaviour of the function $W(\delta)$ defined by (10.16). The solid lines depict the three cases discussed in the text, namely (a) $d_{1}>0$; (b) $d_{1}=0, \sigma_{2} \hat{\tau}_{1}<W^{\prime}\left(0\right.$ and (c) $d_{1}=0$, $\sigma_{2} \hat{\tau}_{1}>W^{\prime}(0)$.

which on substituting from (10.5) can be written as

$$
\delta_{0} \hat{\tau}_{1} \sigma_{2}-d_{1} \sigma_{1}=W\left(\delta_{0}\right)
$$

where

$$
\sigma_{1}=\int_{-\infty}^{\infty} \phi_{0}^{\prime}(s) d s, \quad \sigma_{2}=\int_{-\infty}^{\infty}\left(\phi_{0}^{\prime}(s)\right)^{2} d s
$$

and

$$
W(\delta)=\int_{-\infty}^{\infty} \phi_{0}^{\prime}(s) d s \int_{-\infty}^{s}\left[1-e^{-\delta(s-t)}\right] \phi_{0}^{\prime}(t) d t
$$

the existence of the various integrals being ensured by (10.8).

Despite the apparent complexity of this function, it is easy to establish, see Appendix B, that:

$$
\begin{gathered}
W(0)=0<W(\delta) \leq 2 \quad \text { for all } \quad \delta>0, \\
W(\delta) \rightarrow 2 \quad \text { as } \quad \delta \rightarrow \infty \\
W^{\prime}(\delta)>0 \quad \text { for all } \quad \delta \geq 0,
\end{gathered}
$$

and

$$
W(\delta)<\delta W^{\prime}(0) \text { for all } \delta>0 .
$$

Consequently, $W(\delta)$ behaves as depicted in Figure 10 .

The required value(s) of $\delta_{0}$ are given from (10.14) by the intersection(s) of this curve with the straight line $\delta_{0} \hat{\tau}_{1} \sigma_{2}-d_{1} \sigma_{1}$. Two cases need to be distinguished:

(i) $d_{1}>0$, in which case, since $\sigma_{1}>0$, a unique root always exists. 


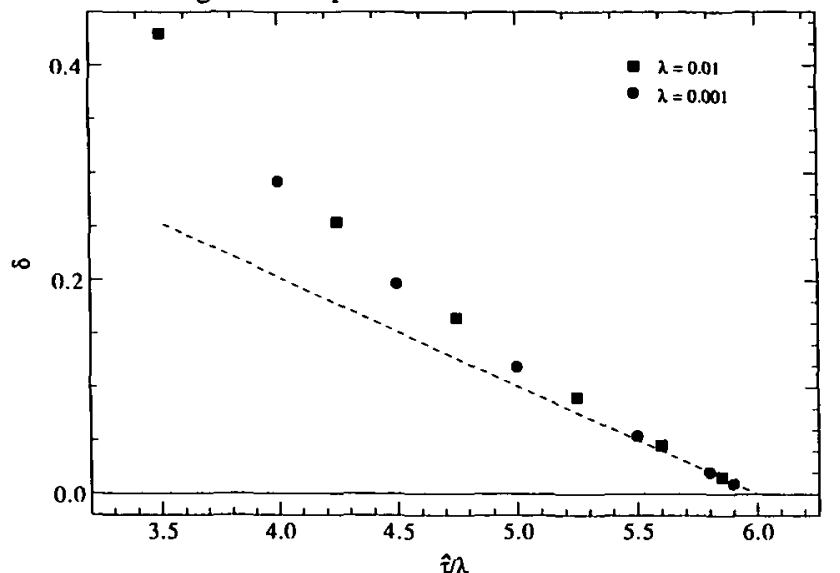

FIGURE 11. Comparison of the asymptotic result $(10.24)$ for $\Delta_{-}=0$ and the indicated values of $\lambda$. $\left(\hat{\tau}_{1} \equiv \hat{\tau} / \lambda\right)$

(ii) $d_{1}=0$, for which a physically relevant non-zero root exists if and only if $\sigma_{2} \hat{\tau}_{1}<W^{\prime}(0)$.

For $\phi^{4}$-theory, substituting for $\phi_{0}^{\prime}(s)$ from $(10.10)$, gives

$$
\sigma_{1}=2, \quad \sigma_{2}=2 / 3
$$

and, see Appendix B,

$$
W^{\prime}(0)=4
$$

Hence if $d_{1}=0$ a physically relevant solution can only exist if $\hat{\tau}_{1}<6$ or in terms of the original parameters $\hat{\tau} / \lambda<6$. While this condition has been derived in the limit $\lambda \rightarrow 0$, it accords exactly with the numerical results.

The behaviour of $\delta_{0}$ in the vicinity of this critical value of $\hat{\tau}_{1}$ follows if we expand $W(\delta)$ to $\mathrm{O}\left(\delta^{2}\right)$. We restrict attention to $\phi^{4}$-theory, for which, see Appendix B,

$$
W(\delta)=4 \delta-\frac{2 \pi^{2} \delta^{2}}{3}+\mathrm{O}\left(\delta^{3}\right) .
$$

Hence, for $\Delta_{-}=0$ and $\hat{\tau}_{1} \rightarrow 6-$,

$$
\delta_{0}=\left(6-\hat{\tau}_{1}\right) / \pi^{2}+O\left(\left(6-\hat{\tau}_{1}\right)^{2}\right)
$$

This prediction is compared with exact numerical values of $\delta$ for several values of $\lambda$ in Figure 11. Agreement is again excellent even for rather large values of $\lambda$.

Since the operator

$$
\mathcal{L}_{0}=\frac{d^{2}}{d s^{2}}+\frac{1}{2}\left(1-3 \tanh ^{2} \frac{1}{2} s\right)
$$


has been exstensively studied in various contexts (see $[40,32,21])$ we can take the $\phi^{4}$ theory calculations a step further and obtain $\phi_{1}$ explicitly. We need the eigenspectrum of $\mathcal{L}_{0}$. This consists [40] of two square integrable functions,

$$
\begin{aligned}
& \eta_{0}=\frac{1}{2} \sqrt{\frac{3}{2}} \operatorname{sech}^{2} \frac{1}{2} s, \\
& \eta_{1}=\frac{1}{2} \sqrt{3} \operatorname{sech} \frac{1}{2} s \tanh \frac{1}{2} s,
\end{aligned}
$$

with eigenvalues $\epsilon_{0}=0$ and $\epsilon_{1}=-3 / 4$, respectively, and a continuum of states,

$$
\tilde{\eta}_{k}=\mathcal{N}_{k}^{-1} e^{i k s / 2}\left(1+k^{2}+3 i k \tanh \frac{1}{2} s-3 \tanh ^{2} \frac{1}{2} s\right), \quad-\infty<k<\infty,
$$

with eigenvalues

$$
\epsilon_{k}=-1-k^{2} / 4
$$

If the normalization factors $\mathcal{N}_{k}$ are taken to be

$$
\mathcal{N}_{k}=2 \sqrt{\pi\left(k^{2}+4\right)\left(k^{2}+1\right)},
$$

these states satisfy

$$
\left\langle\tilde{\eta}_{k^{\prime}}, \tilde{\eta}_{k}\right\rangle=\int_{-\infty}^{\infty} \tilde{\eta}_{k^{\prime}}^{*}(s) \tilde{\eta}_{k}(s) d s=\delta\left(k^{\prime}-k\right),
$$

where $\delta(k)$ is the Dirac delta function and * denotes complex conjugation.

Since this set of eigenstates is complete we can use them as a basis to expand the function $\bar{R}(s)$ appearing in (10.11). Some care with convergence is necessary since

$$
\bar{R}(s) \rightarrow\left\{\begin{array}{lll}
d_{1} & \text { as } & s \rightarrow-\infty \\
d_{1}+2 & \text { as } & s \rightarrow+\infty
\end{array}\right.
$$

However, these problems can be circumvented, see Appendix C, with the result that

$$
\bar{R}(s)=r_{1} \eta_{1}(s)-2 \sqrt{\pi} \tilde{\eta}_{0}(s)+\int_{-\infty}^{\infty} \hat{r}(k) \tilde{\eta}_{k}(s) d k,
$$

where, in view of the solvability criterion, we have omited the term involving $\eta_{0} \propto \phi_{0}^{\prime}$, the coefficient $r_{1}$ is given by (C.13) of Appendix C,

$$
\hat{r}(k) \sim \frac{1}{i k \sqrt{\pi}}+\mathrm{O}(1) \quad \text { as } \quad k \rightarrow 0,
$$


and, in the integral over $k$, we require $\operatorname{Im} k=0^{-}$for convergence. Assuming a similar expansion for $\phi_{1}$ allows the required expansion coefficients to be written down by inspection. It is convenient to write the resulting expansion as

$$
\phi_{1}(s)=C_{0} \eta_{0}(s)-\bar{R}(s)-\frac{1}{3} r_{1} \eta_{1}(s)+\int_{-\infty}^{\infty} \frac{k^{2} \hat{r}(k)}{4+k^{2}} \tilde{\eta}_{k}(s) d k,
$$

where $C_{0}$ is an arbitrary constant that can be incorporated (since $\eta_{0} \propto \phi_{0}^{\prime}$ ) as a shift of the arbitrary position of the interface. In view of (10.34) the integral vanishes as $s \rightarrow \pm \infty$ so that, recalling (10.32), we have

$$
\phi_{1}(s) \rightarrow\left\{\begin{array}{lll}
-d_{1} & \text { as } & s \rightarrow-\infty, \\
-d_{1}-2 . & \text { as } & s \rightarrow+\infty
\end{array}\right.
$$

in accord with (10.7).

\section{Summary and concluding comments}

In the preceding sections of this paper the existence and selection of steady-state of travelling planar fronts in a set of typical phase field equations of solidification have been investigated numerically and analytically in certain tractable limits. These investigations give considerable support to the conjecture first enunciated in Section 8, namely that solutions to the phase field equations corresponding to steady-state planar fronts exist only for a unique velocity $c$; such a solution is moreover unique except for translation. This behaviour is in marked contrast to the situation in conventional Stefan-type models in which travelling fronts exist for all velocities. ${ }^{12}$

The precise value of the steady-state velocity depends upon the various material parameters which enter the phase field equations. If $\tau$ is order $\epsilon$, then matched asymptotic expansions in $\epsilon$ yield $c(\epsilon)=c_{0}+c_{1} \epsilon+\mathrm{O}\left(\epsilon^{2}\right)$; recall Section 7. If $\tau$ and $\epsilon$ are both arbitrary parameters, then $c(\epsilon, \tau)=\delta_{c} / \epsilon$, where the behaviour of the parameter $\delta_{c}$ is illustrated in Figure 8 of Section 8 . A particularly striking feature is the behaviour for $\Delta_{-}=0$ in which case a critical value of the material parameter $\lambda$, related to the latent heat, appears to exist. Only if $\lambda$ exceeds this critical value do travelling waves appear to exist.

We have been able to substantiate much of the behaviour illustrated in Figure 8 by considering the phase field equations in the limit $\lambda \rightarrow 0$. In particular, the behaviour for $\Delta_{-}=0$ can be analytically demonstrated in an expansion in which both $\hat{\tau} \equiv \tau / \epsilon^{2}$ and $\Delta_{-}$are taken to be $O(\lambda)$ as $\lambda$ tends to zero; recall Section 10 .

\footnotetext{
${ }^{12}$ In both the phase field equations and the Stefan model, steady-state front solutions exist only for a specific combination of boundary data. In the Stefan problem this is the so called "Stefan number unity condition", while in the phase field equations the analogous condition is that of $(4.10)$.
} 
The analysis of Section 10 is important for a further reason. A common feature of both the $\epsilon$-expansions of Section 7 and the $\lambda$-expansions of Sections 9 and 10 is the existence of a solvability criterion for the first order corrections. Satisfaction of this criterion is the fundamental mathematical origin of velocity selection. However, the solvability criterion is only a necessary condition and need not be sufficient. Only for the special limit analysed in Section $\mathbf{1 0}$ are we able to explicitly construct, by a spectral method, the first-order corrections and confirm that they satisfy the relevant boundary conditions.

Indeed, it is possible to exhibit a case in which the solvability criterion implies a solution, but no solution, in fact, exists. This example arises if, as suggested by Figure 8 , we try to analyse (9.1) with $\Delta_{-}=0$ by expanding in $\delta$. Assuming that we can expand

$$
\phi=\phi_{0}+\delta \phi_{1}+\mathrm{O}\left(\delta^{2}\right)
$$

we find that $\phi_{0}$ satisfies

$$
\phi_{0}{ }^{\prime \prime}+f\left(\phi_{0}\right)=0
$$

The natural boundary conditions are $\phi_{0} \rightarrow \pm 1$ as $s \rightarrow \pm \infty$. Hence within $\phi^{4}$-theory we again recover the familiar kink solution. Proceeding to first order in $\delta$ leads again to the solvability criterion

$$
\hat{\tau} \int_{-\infty}^{\infty}\left(\phi_{0}^{\prime}(\xi)\right)^{2} d \xi=\lambda
$$

For $\phi^{4}$-theory this condition reduces to the relation $\hat{\tau} / \lambda=6$, which accords precisely with the numerical estimate of the critical value of $\lambda$. There is, however, a problem. From the boundary conditions applied to the full function $\phi$ and those applied to $\phi_{0}$ we find that $\phi_{1}$ cannot remain bounded. Hence, no acceptable solution exists. This cautionary tale suggests that a rigorous confirmation of our conjecture would be desirable. We have not been able to construct such a proof and leave it as an open question.

Throughout this paper we have referred at times to related work, notably the work of Wilder [39] who anticipated for the special case of $\phi^{4}$-theory many of the conclusions of Section 7. The other particularly relevant recent work is that by Caginalp and Nishiura [9] in which they were able to establish the existence of travelling wave solutions in the form that we have conjectured but in a different distinguished limit to any considered here.

This limit, in our notation, can be obtained by returning to the basic free energy functional(2.2) and introducing an additional (and arbitrary) parameter $a^{-1}$ multiplying the function $\Psi(\phi)$. The effect of $a$ can be seen if we repeat the equilibrium calculation of Section 3 for the surface tension; the key result-specialising to $\phi^{4}$-theory-(3.5) 
becoming

$$
\sigma / k_{B} T_{M}=\frac{2}{3} \sqrt{\frac{K}{a}} \propto \epsilon / \sqrt{a} .
$$

All the cases analysed in this paper, corresponding to $a=1$, are characterised by a surface tension that vanishes as $\epsilon \rightarrow 0$, a limit in which essentially a standard Stefan problem is recovered. Caginalp and Nishiura [9] assume instead that $a=O\left(\epsilon^{2}\right)$ so that $\sigma \rightarrow \sigma_{0}$ as $\epsilon \rightarrow 0$, where $\sigma_{0}$ is taken to be the physical surface tension. Caginalp and Nishiura were then able to prove that in this limit travelling wave solutions to the phase field equations exist and that they converge to the corresponding solutions of a modified Stefan problem incorporating as boundary conditions on the free surface the Gibbs-Thomson correction and an additional kinetic correction. The one-dimensional version of this model has been discussed by Dewynne et al. [12] and is known to exhibit a unique velocity determined by the undercooling. While the Caginalp/Nishiura result is mathematically pleasing it actually begs the more interesting physical question of how the degeneracy present in the absence of surface tension, that is, in a simple Stefan problem, is removed by the inclusion of surface tension.

This question has been the focus of much attention in higher dimensions, particularly with regard to selection of the primary morphology and velocity of dendrites $[27,22,30]$. In this context the key question has been: Does the inclusion of surface tension remove the degeneracy present in the so-called Ivantsov [20, 25] families of parabolic (in two dimensions) or paraboloidal (in three dimensions) needle crystals? Our one-dimensional results have little bearing on this question except as an example of how the introduction of a finite thickness to the interfacial region and a qualitatively correct thermodynamic description can resolve degeneracies that arise in simpler and physically less complete models. While one can easily show that for $\epsilon=\tau=0$ the phase-field equations in two dimensions possess one parameter Ivantsov-like solutions, the matched asymptotic analysis to include the effects of finite $\epsilon$ and $\tau$ is technically difficult and will be discussed elsewhere. [34]

Numerical results by one of us (Singleton) and independent work by Kobayashi [24] suggest that the phase field equations in two or three dimensions do exhibit structures reminiscent of dendrites. In particular, the numerical simulations reveal solutions for which the level sets of the phase field exhibit a parabolic tip moving at a uniform velocity behind which trail side branch-like structures. This has been most spectacularly demonstrated in recent work by Kobayashi [24] on a modified system of phase field equations. While it appears difficult to tune the material parameters to realistic values, this could be simply an artifact of the simplicity of underlying thermodynamic formulations that have been used to date. We intend to return to this question in a subsequent paper.

With regard to the one-dimensional results that we have discussed in this paper, we believe that they are typical of the behaviour that would occur in more realistic 
systems, which are either based on more realistic thermodynamic descriptions or allow for the approximations made in the derivation of the phase field equations presented in Section 2. While the reduction to a third-order system that underpins the analysis of Section 7 is probably special, those considerations appear to rely on fundamental aspects of the stability of the two relevant fixed points. It is difficult to see how these features could be significantly modified without at the same time having unphysical effects on the thermodynamics of the bulk phases. Nevertheless, this question should be explicitly checked and the numerical method developed in Section 7 is capable of handling more complex situations.

A number of other questions suggest themselves. The stability of a planar interface moving in higher dimensions is one. As is well-known [25] sharp interface models exhibit a morphological instability—the Mullins/Sekerka instability [29]—which is ultimately controlled by the effect of surface tension. It would also be interesting to know if violation of the special condition (4.10) on the boundary data gives rise to similarity solutions or how the similarity solutions of the Stefan problem [19] are modified by the presence of a finite interface. Some work [33] is possible on these questions and will be reported elsewhere.

\section{Acknowledgements}

The authors would like to thank Professors J. Langer, G. Caginalp and G. Meyer and $\mathrm{Mr} \mathrm{A}$. Roberts for a number of informative discussions. This work was supported by the Australian Research Council.

\section{Appendix A. Numerical evaluation of $K(p, q)$}

The hypergeometric function ${ }_{3} F_{2}$ appearing in (9.22) for $K(p, q)$ is defined by [13]

$$
\begin{aligned}
{ }_{3} F_{2}\left(a_{1}, a_{2}, a_{3} ; b_{1}, b_{2} ; z\right) & \\
= & \frac{\Gamma\left(b_{1}\right) \Gamma\left(b_{2}\right)}{\Gamma\left(a_{1}\right) \Gamma\left(a_{2}\right) \Gamma\left(a_{3}\right)} \sum_{m=0}^{\infty} \frac{\Gamma\left(a_{1}+m\right) \Gamma\left(a_{2}+m\right) \Gamma\left(a_{3}+m\right)}{\Gamma\left(b_{1}+m\right) \Gamma\left(b_{2}+m\right) m !} z^{m} .
\end{aligned}
$$

Provided $\operatorname{Re}\left(\sum b_{i}-\sum a_{i}\right)>0$ this series is convergent for $|z| \leq 1$. In the case of (9.22) this condition corresponds to the condition $p-q<1$ or $(\hat{\tau}-1) \delta_{0}<2 \alpha_{0}$, which does not appear to be limiting for physically relevant values of the parameters. Hence we can represent $K(p, q)$ by the convergent series:

$$
K(p, q)=\frac{1+p}{1+q} \frac{\pi p}{\sin \pi p} \sum_{m=1}^{\infty} t_{m}
$$


where

$$
t_{m}=\frac{2 \Gamma(2+q) \Gamma(1+p+m)}{m(m+1) \Gamma(2+p) \Gamma(1+q+m)}
$$

Note that

$$
\begin{aligned}
& t_{m+1}=\frac{m(1+p+m) t_{m}}{(m+2)(1+q+m)}, \quad t_{1}=1, \\
& t_{m}=\frac{2 \Gamma(2+p)}{\Gamma(2+q)} m^{-2-(q-p)}\left\{1+\mathrm{O}\left(m^{-1}\right)\right\} .
\end{aligned}
$$

Hence the sequence

$$
T_{M}(p, q)=\sum_{m=1}^{M} t_{m}, \quad M=1,2, \ldots
$$

converges as

$$
T_{M}=T_{\infty}+O\left(M^{-1-(q-p)}\right) \quad \text { as } \quad M \rightarrow \infty
$$

with

$$
K(p, q)=\frac{1+p}{1+q} \frac{\pi p}{\sin \pi p} T_{\infty} .
$$

Accurate estimates of $T_{\infty}$ can be obtained by the use of a number of standard acceleration algorithms. We chose the $\theta$-algorithm [3]:

$$
\begin{aligned}
\theta_{n}^{(k)} & =\theta_{n}^{(k-1)}+1 / \Delta T_{n}^{(k)}, \\
T_{n}^{(k+1)} & =T_{n+1}^{(k)}+\Delta T_{n+1}^{(k)} \theta_{n+1}^{(k)} / \Delta^{2} \theta_{n}^{(k)},
\end{aligned}
$$

with $\theta_{n}^{-1}=0$ and the alternating $\epsilon$-algorithm [2]:

$$
\begin{aligned}
\epsilon_{n}^{(2 k+1)} & =\alpha_{k} \epsilon_{n}^{(2 k-1)}+1 / \Delta \epsilon_{n}^{(2 k)}, \\
\epsilon_{n}^{(2 k+2)} & =\epsilon_{n}^{(2 k)}+1 / \Delta \epsilon_{n-1}^{(2 k+1)}, \\
T_{n}^{(k)} & =\epsilon_{n}^{4 k},
\end{aligned}
$$

where $\epsilon_{n}^{(-1)}=0, \epsilon_{n}^{(0)}=T_{n}^{(0)}$ and $\alpha_{k}=\left[(-1)^{k}-1\right] / 2$. In (A.9) to (A.13), $T_{n}^{(0)} \equiv K_{n}$ and $\Delta x_{n}=x_{n+1}-x_{n}$.

Both these algorithms are known $[3,2]$ to accelerate sequences of the form of (A.7) in the sense that if

$$
T_{M}=T_{\infty}+A M^{-\lambda}\left\{1+\mathrm{O}\left(M^{-1}\right)\right\}
$$

then the accelerated sequence $T_{M}^{(1)}$ converges as

$$
T_{M}^{(1)}=T_{\infty}+O\left(M^{-\lambda-2}\right)
$$

Typical results for $\hat{\tau}=0.4$, and $\Delta_{-}=0.05$ (and hence $\delta_{0}=0.378867 \ldots$, $p=0.152130 \ldots$ and $q=0.380326 \ldots$ ) are shown in Table 1 . These results lead to the confident estimate that for these parameter values $\delta_{1}=4.348856 \ldots$. 
TABLE 1. Typical acceleration of the sequence $T_{M}$.

\begin{tabular}{|c|c|c|c|c|c|}
\hline \multirow{3}{*}{$\frac{M}{1}$} & \multirow{3}{*}{$\frac{T_{M}}{1.0000000}$} & \multicolumn{4}{|c|}{ Accelerations } \\
\hline & & \multicolumn{2}{|c|}{ alt. $\epsilon$-algorithm } & \multicolumn{2}{|c|}{$\theta$-algorithm } \\
\hline & & 1.7778506 & 1.7774599 & 1.7732855 & 1.7780120 \\
\hline 2 & 1.3013776 & 1.7776618 & 1.7774601 & 1.7759578 & 1.7774535 \\
\hline 3 & 1.4418938 & 1.7775765 & 1.7774602 & 1.7767682 & 1.7774580 \\
\hline 4 & 1.5218114 & 1.7775328 & 1.7774602 & 1.7770898 & 1.7774593 \\
\hline 5 & 1.5728301 & 1.7775083 & 1.7774602 & 1.7772407 & 1.7774598 \\
\hline 6 & 1.6079686 & 1.7774936 & 1.7774602 & 1.7773203 & 1.7774600 \\
\hline 7 & 1.6335077 & 1.7774843 & 1.7774602 & 1.7773659 & 1.7774601 \\
\hline 8 & 1.6528305 & 1.7774781 & & 1.7773939 & 1.7774601 \\
\hline 9 & 1.6679128 & 1.7774738 & & 1.7774119 & 1.7774602 \\
\hline 10 & 1.6799815 & 1.7774708 & & 1.7774240 & \\
\hline 11 & 1.6898371 & 1.7774686 & & 1.7774325 & \\
\hline 12 & 1.6980227 & & & 1.7774385 & \\
\hline 13 & 1.7049193 & & & & \\
\hline 14 & 1.7108015 & & & & \\
\hline 15 & 1.7158721 & & & & \\
\hline
\end{tabular}

Appendix B. The function $W(\delta)$

In this appendix we establish a number of results, cited in the text, concerning the function

$$
W(\delta)=\int_{-\infty}^{\infty} \phi_{0}^{\prime}(s) d s \int_{-\infty}^{s}\left[1-e^{-\delta(s-t)}\right] \phi_{0}^{\prime}(t) d t,
$$

which was originally defined in (10.16). The function $\phi_{0}(s)$ satisfies (10.4).

Since $\phi_{0}^{\prime}(s)$ is positive, recall the discussion in the main text between (10.8) and (10.9), clearly $W(0)>0$. In addition, since $1>1-e^{-\delta(t-s)}>0$ for $t>s$ and $\lim _{s \rightarrow \pm \infty} \phi_{0}(s)= \pm 1$,

$$
0<W(\delta)<\int_{-\infty}^{\infty} \phi_{0}^{\prime}(s) d s \int_{-\infty}^{s} \phi_{0}^{\prime}(t) d t=2
$$

The right-hand side of this inequality is also $\lim _{\delta \rightarrow \infty} W(\delta)$. 
If the upper bound on $1-e^{-\delta(t-s)}$ is sharpened to

$$
1-e^{-\delta(t-s)}<\delta(t-s), \quad t>s,
$$

we obtain

$$
W(\delta)<\delta W^{\prime}(0)
$$

where

$$
W^{\prime}(\delta)=\int_{-\infty}^{\infty} \phi_{0}^{\prime}(s) d s \int_{-\infty}^{s}(s-t) e^{-\delta(s-t)} \phi_{0}^{\prime}(t) d t
$$

is positive for all $\delta \geq 0$ by the positivity of $\phi_{0}^{\prime}$.

For $\phi^{4}$-theory, $\phi_{0}^{\prime}=\frac{1}{2} \operatorname{sech}^{2} \frac{1}{2} s$, and hence

$$
W(\delta)=\int_{-\infty}^{\infty} \operatorname{sech}^{2} s d s \int_{-\infty}^{s}\left[1-e^{-2 \delta(s-t)}\right] \operatorname{sech}^{2} t d t .
$$

Setting $\operatorname{sech}^{2} t=d(1+\tanh t) / d t$ and integrating by parts in the inner integral yields

$$
W(\delta)=2 \delta \int_{-\infty}^{\infty} \operatorname{sech}^{2} s d s \int_{-\infty}^{s} e^{-2 \delta(s-t)}(1+\tanh t) d t=2 \delta K(0, \delta),
$$

where $K(p, q)$ is defined in (9.20). Hence from (9.22),

$$
W(\delta)=\frac{2 \delta}{1+\delta}{ }_{3} F_{2}(1,1,2 ; 2+\delta, 3 ; 1),
$$

which, on introducing the series representation of ${ }_{3} F_{2}$, recall (A.1), gives

$$
W(\delta)=4 \delta \Gamma(1+\delta) \sum_{m=1}^{\infty} \frac{\Gamma(m+1)}{\Gamma(m+1+\delta)} \cdot \frac{1}{m(m+1)} .
$$

Since this series is absolutely convergent, we can rearrange the summand by writing

$$
\frac{1}{m(m+1)}=\frac{1}{m}-\frac{1}{m+1}
$$

to obtain

$$
W(\delta)=4 \delta-4 \delta^{2} \Gamma(1+\delta) \sum_{m=1}^{\infty} \frac{\Gamma(m)}{\Gamma(m+\delta)} \cdot \frac{1}{m(m+\delta)} .
$$

The result $W^{\prime}(0)=4$ quoted in the text follows immediately, while expanding the sum to leading order in $\delta$ gives

$$
\begin{aligned}
W(\delta) & =4 \delta-4 \delta^{2} \sum_{m=1}^{\infty} \frac{1}{m^{2}}+O\left(\delta^{3}\right) \\
& =4 \delta-\frac{2 \pi^{2} \delta^{2}}{3}+O\left(\delta^{3}\right)
\end{aligned}
$$




\section{Appendix C. Expansion of $\bar{R}(s)$}

In this appendix we summarize the expansion of the function $\bar{R}(s)$ that appears on the right hand side of (10.5), in terms of the eigenfunctions, (10.26) to (10.28), of the operator $\mathcal{L}_{0}$. It is convenient to write

$$
\bar{R}(s)=d_{1}-\delta_{0} \hat{\tau}_{1} \phi_{0}^{\prime}(s)+H(s),
$$

where

$$
H(s)=\int_{-\infty}^{2 s}\left[1-e^{-\delta_{0}(s-2 t)}\right] \operatorname{sech}^{2} t d t .
$$

Expanding the integrand appropriately leads to the convergent series representations:

$$
H(s)=2 \delta_{0} \sum_{r=1}^{\infty} \frac{(-)^{r-1} e^{r s}}{r+\delta_{0}}
$$

for $s \leq 0$ and

$$
H(s)=2-\frac{2 \pi \delta_{0}}{\sin \pi \delta_{0}} e^{-\delta_{0} s}+2 \delta_{0} \sum_{r=1}^{\infty} \frac{(-)^{r-1} e^{-r s}}{r-\delta_{0}}
$$

for $s \geq 0$.

Since the parameter $\delta_{0}$ is determined by the solvability condition, $\left\langle\bar{R}, \eta_{0}\right\rangle=0$, and $\phi_{0}^{\prime} \propto \eta_{0}$, we can omit the term involving $\eta_{0}$ in the required expansion. The convergence problem alluded to in the main text arises because $\bar{R}(s)$ has non-zero limits as $s \rightarrow \pm \infty$. From (C.3) and (C.4),

$$
\bar{R}(s)=\left\{\begin{array}{lll}
d_{1}+O\left(e^{s}\right) & \text { as } & s \rightarrow-\infty \\
d_{1}+2+O\left(e^{-s \min \left(1, \delta_{0}\right)}\right) & \text { as } & s \rightarrow+\infty .
\end{array}\right.
$$

Hence we write

$$
\bar{R}(s)=-2 d_{1} \sqrt{\pi} \tilde{\eta}_{0}(s)+2 \Theta(s)+\bar{R}_{1}(s),
$$

where $\Theta(s)$ is the Heaviside step function,

$$
\Theta(s)= \begin{cases}0 & \text { for } s<0 \\ 1 & \text { for } s>0\end{cases}
$$

and

$$
\tilde{\eta}_{0}(s)=\frac{1}{4 \sqrt{\pi}}\left(1-3 \tanh ^{2} \frac{1}{2} s\right) \rightarrow-\frac{1}{2 \sqrt{\pi}} \quad \text { as } \quad s \rightarrow \pm \infty,
$$

is the eigenfunction of $\mathcal{L}_{0}$ with eigenvalue -1 . The function $\bar{R}_{1}(s)$ now tends exponentially fast to zero as $s \rightarrow \pm \infty$. Consequently, the coefficients

$$
\bar{a}_{k}=\int_{-\infty}^{\infty} \tilde{\eta}_{k}^{*}(s) \bar{R}_{1}(s) d s
$$


exist and are finite for all $k$. For our purposes it suffices to note that, given the exponential decay of $\bar{R}_{1}(s), \bar{a}_{k}=\mathrm{O}(1)$ as $k \rightarrow 0$. Similarily, it suffices to observe that

$$
\begin{aligned}
\int_{-\infty}^{\infty} \tilde{\eta}_{k}^{*}(s) \Theta(s) d s & =\frac{1}{2 \sqrt{\pi}} \int_{0}^{\infty} e^{-i k s}\left[1-3 \tanh ^{2} s+\mathrm{O}(k)\right] d s \\
& =\frac{1}{i k \sqrt{\pi}}+\mathrm{O}(1) \quad \text { as } \quad k \rightarrow 0,
\end{aligned}
$$

where to ensure convergence we assume $\operatorname{Im} k=0^{-}$. Combining these observations yields the assertion (10.34) for the coeficients $\hat{r}(k)$.

It remains to compute the coefficient $r_{1}$ of the term involving $\eta_{1}$. From (C.6)

$$
r_{1}=\bar{r}_{1}+2 \int_{0}^{\infty} \eta_{1}(s) d s=\bar{r}_{1}+2 / \sqrt{3}
$$

where

$$
\bar{r}_{1}=\int_{-\infty}^{\infty} \eta_{1}(s) \bar{R}_{1}(s) d s
$$

Hence on combining (C.1), (C.3), (C.4) and (C.6) we obtain

$$
r_{1}=\frac{2}{\sqrt{3}}-\frac{2 \pi \delta_{0}}{\sin \pi \delta_{0}} B\left(\delta_{0}\right)+4 \delta_{0}^{2} \sum_{r=1}^{\infty} \frac{(-)^{r-1}}{r^{2}-\delta_{0}^{2}} B(r),
$$

where

$$
B(\gamma)=\int_{0}^{\infty} \eta_{1}(s) e^{-\gamma s} d s
$$

Substituting (10.27) for $\eta_{1}$ allows this final integral to expressed [15, page 256, \#3.54] in terms the logarithmic derivative of the gamma function. Explicitly,

$$
B(\gamma)=\sqrt{3}-2 \gamma \sqrt{3}\left[\psi\left(\frac{\gamma}{2}+\frac{3}{4}\right)-\psi\left(\frac{\gamma}{2}+\frac{1}{4}\right)\right] .
$$

Since $B(\gamma)=\mathrm{O}(1 / \gamma)$ as $\gamma \rightarrow \infty$, the series in (C.13) converges absolutely.

\section{References}

[1] M. J. Ablowitz and A. Zeppetella, "Explicit solutions of Fisher's equation for a special wave speed", Bull. Math. Biol. 41 (1979) 835-840.

[2] M. N. Barber and C. J. Hamer, "Extrapolation of sequences using a generalized epsilon-algorithm", J. Austral. Math. Soc. Ser. B 23 (1982) 229-240.

[3] C. Brezinski, "Accélération de suites à convergence logarithmique”, C. R. Acad. Sci. Paris Sér. A-B 273 (1971) A727-A730. 
[4] G. Caginalp, "An analysis of a phase field model of a free boundary", Arch. Rational Mech. Anal. 92 (1986) 205-245.

[5] G. Caginalp, "Stefan and Hele-Shaw type models as asymptotic limits of phase-field equations", Phys. Rev. A 39 (1989) 5887-5896.

[6] G. Caginalp and X. Chen, "Phase field equations in the singular limit of sharp interface problems", in On the evolution of phase boundaries (eds. M. E. Gurtin and G. B. McFadden), (Springer-Verlag, New York, 1992) 1-15.

[7] G. Caginalp and P. C. Fife, "Dynamics of layered interfaces arising from phase boundaries", SIAM J. Appl. Math. 48 (1988) 506-518.

[8] G. Caginalp and J. Jones, A phase field model: Derivation and new interface relation (1991).

[9] G. Caginalp and Y. Nishiura, "The existence of travelling waves for phase field equations and convergence to sharp interface models in the singular limit", Quart. J. Appl. Math. 49 (1991) $147-162$.

[10] J. W. Cahn and J. E. Hilliard, "Free energy of a non-uniform system. I. Interfacial free energy", J. Chem. Phys. 28 (1957) 258-267.

[11] J. B. Collins and H. Levine, "Diffuse interface model of diffusion-limited crystal growth", Phys. Rev. B 31 (1985) 6119-6122.

[12] J. N. Dewynne, S. D. Howison, J. R. Ockendon and W. Xie, "Asymptotic behavior of solutions to the Stefan problem with a kinetic condition at the free boundary", J. Austral. Math. Soc. Ser. B 31 (1989) 81-96.

[13] A. Erdélyi (ed.), Higher transcendental functions, Volume 1 (Academic Press, New York, 1953).

[14] P. C. Fife and J. B. McLeod, "The approach of solutions of nonlinear diffusion equations to travelling front solutions", Arch. Rational Mech. Anal. 65 (1977) 335-361.

[15] I. S. Gradshteyn and I. M. Ryzhik, Table of integrals, series and products (Academic Press, London, 1980).

[16] P. S. Hagan, "The instability of non-monotonic wave solutions of parabolic equations", Stud. Appl. Math. 64 (1981) 57-88.

[17] P. S. Hagan, "Traveling waves and multiple traveling wave solutions of parabolic equations", SIAM J. Math. Anal. 13 (1982) 717-738.

[18] G. Hall and J. M. Watts, Modern methods for ordinary differential equations (Clarendon Press, Oxford, 1976).

[19] S. D. Howison, J. R. Ockendon and A. A. Lacey, "Singularity development in moving boundary problems", Quart. J. Mech. Appl. Math. 38 (1985) 343-360.

[20] G. P. Ivantsov, "Temperature field around a spheroidal, cylindrical and acicular crystal growing in a supercooled melt", in Doklady Akad. Nauk SSSR, Volume 58, (originally published in Russian, reprinted and translated to English in Ref. [30], p.243), 1947) 567.

[21] D. Jasnow, "Critical phenomena at interfaces", Rep. Prog. Phys. 47 (1984) 1059-1132.

[22] D. A. Kessler, J. Koplik and H. Levine, "Pattern selection in fingered growth phenomena", Adv. Phys. 37 (1988) 255-339.

[23] J. Kevorkian and J. D. Cole, Perturbation methods in applied mathematics (Springer-Verlag, New York, 1981).

[24] R. Kobayashi, "Simulations of three-dimensional dendrites", 1991, preprint.

[25] J. S. Langer, "Instabilities and pattern formation in crystal growth", Rev. Mod. Phys. 52 (1980) 1-28.

[26] J. S. Langer, "Models of pattern formation in first-order phase transitions", in Directions in condensed matter physics (eds. G. Grinstein and G. Mazenko), (World Scientific, Singapore, 1986) 165-186.

[27] J. S. Langer, "Lectures in the theory of pattern formation", in Chance and matter (eds. J. Souletie, J. Vanimenus and R. Stora (Les Houches XIVI 1986)), (North Holland, Amsterdam, 1987) 629- 
711.

[28] S. Lefschetz, Differential equations: Geometric theory (Interscience Publishers, New York, 1957).

[29] W. W. Mullins and J. Sekerka, "Morphological stability of a particle growing by diffusion or heat flow", J. Appl. Phys. 34 (1963) 323-329.

[30] P. Pelce, Dynamics of curved fronts (Academic Press, New York, 1989).

[31] O. Penrose and P. C. Fife, "Thermodynamically consistent models of phase-field type for the kinetics of phase transitions", Physica D 43 (1990) 44-62.

[32] R. Rajaraman, "Some non-perturbative semi-classical methods in quantum field theory", Phys. Rep. 21C (1975) 228-313.

[33] A. Roberts, unpublished.

[34] A. Roberts and M. N. Barber, unpublished.

[35] J. S. Rowlinson and B. Widom, Molecular theory of capillarity (Clarendon Press, Oxford, 1982).

[36] L. I. Rubinstein, "The Stefan problem", Am. Math. Soc. Transl. 27 (1971).

[37] M. D. Van Dyke, Perturbation methods in fluid mechanics (Academic Press, New York, 1964).

[38] A. A. Wheeler, W. J. Boettinger and G. B. McFadden, "A phase-field model for isothermal phase transitions in binary alloys", Phys. Rev. A 45 (1992) 7424-7439.

[39] J. W. Wilder, "Travelling wave solutions for interfaces arising from phase boundaries based on a phase field model", Quart. J. Appl. Math. 49 (1991) 333-350.

[40] J. Zittartz, "Microscopic approach to interfacial structure in Ising-like ferromagnets", Phys. Rev. 154 (1967) 529-534. 